\title{
Instability, Intermittency and Multiscaling in Discrete Growth Models of Kinetic Roughening
}

\author{
C. Dasguptat, J. M. Kim**, M. Dutta and S. Das Sarma \\ Department of Physics, University of Maryland, College Park, Maryland 20742-4111.
}

(August 6, 2018)

\begin{abstract}
We show by numerical simulations that discretized versions of commonly studied continuum nonlinear growth equations (such as the Kardar-Parisi-Zhang equation and the Lai-Das Sarma equation) and related atomistic models of epitaxial growth have a generic instability in which isolated pillars (or grooves) on an otherwise flat interface grow in time when their height (or depth) exceeds a critical value. Depending on the details of the model, the instability found in the discretized version may or may not be present in the truly continuum growth equation, indicating that the behavior of discretized nonlinear growth equations may be very different from that of their continuum counterparts. This instability can be controlled either by the introduction of higher-order nonlinear terms with appropriate coefficients or by restricting the growth of pillars (or grooves) by other means. A number of such "controlled instability" models are studied by simulation. For appropriate choice of the parameters used for controlling the instability, these models exhibit intermittent behavior, characterized by multiexponent scaling of height fluctuations, over the time interval during which the instability is active. The behavior found in this regime is very similar to the "turbulent" behavior observed in recent simulations of several one- and two-dimensional atomistic models of epitaxial growth.
\end{abstract}

61.50.Cj, 68.55.Bd, 05.70.Ln, 64.60.Ht

\section{INTRODUCTION}

In recent years, much attention has been focused on the problem of kinetic surface roughening associated with the nonequilibrium dynamics of growing interfaces [1] 2]. A number of simple models of epitaxial growth have been proposed and studied [3 9] analytically and numerically, revealing a rich variety of interesting phenomena. One such phenomenon for which no explanation is currently available is the multi-exponent scaling ("multiscaling" in short) of height fluctuations found [8] in recent simulations [8 10] of a class of one-dimensional (1d) limited-mobility models of epitaxial growth. This phenomenon is particularly interesting because it exhibits a striking similarity [8] to the intermittent multiscaling of velocity fluctuations in fully developed fluid turbulence [11].

This paper describes the results of a numerical investigation of the origin of this interesting multiscaling behavior. Our study shows that the multiscaling found in these models is closely related to an instability of discretized versions of commonly studied nonlinear growth equations. In this instability, isolated structures (such as pillars or grooves) on a flat interface tend to grow in time if the "size" of the structure (i.e. height of a pillar or depth of a groove) exceeds a critical value. We show that this instability is the cause of numerical difficulties encountered in earlier work [12,13] on numerical integration of discretized growth equations. These difficulties were usually attributed to "numerical artifacts" in previous studies. In contrast, we show that these numerical difficulties are the consequence of a genuine instability intrinsic to the discretized growth equations. This instability is found to be "generic" to a large class of discretized growth equations with nonlinearities. In particular, we find that this instability is present in $1 \mathrm{~d}$ and $2 \mathrm{~d}$ versions of the conserved fourth-order growth equation introduced by Lai and Das Sarma (LD) [4] and by Villain [5], and also in the 1d Kardar-Parisi-Zhang (KPZ) equation [3] with or without noise. Since the 1d continuum KPZ equation without noise, which is exactly solvable via a mapping to the diffusion equation by a ColeHopf transformation [2], does not have any instability, our results lead to the important conclusion that the behavior of discretized nonlinear growth equations may be very different from that of the corresponding truly continuum versions. Whether this instability occurs or not during the time evolution of a system started from a flat initial state is determined by the nature of the dynamic scaling exhibited by the system. We find that this instability is inevitable at sufficiently long times in models which exhibit "anomalous" dynamic scaling [14] (provided the system size is sufficiently large to prevent saturation), whereas models exhibiting conventional scaling show this instability only if the value of a dimensionless coupling constant (defined below in terms of the values of the parameters in the original growth equation and the length scale of discretization) exceeds a non-zero critical value. A similar instability is found in an atomistic model [7] which is believed to provide an exact discrete representation of the continuum LD growth equation. 
Next, we show that this instability can be controlled by introducing higher-order nonlinear terms with appropriate coefficients. These higher-order terms cut off the growth of pillars/grooves at large values of the height/depth. The instability in atomistic growth models can also be controlled by modifying the deposition rule in a way that restricts the growth of the height of a pillar or the depth of a groove. We find that such models with controlled instability exhibit deviations from simple scaling in the time interval during which the instability is operative. If the parameter(s) used in the control of the instability is (are) chosen properly, then the behavior in this regime is found to be very similar to the multiscaling observed in simulations [8 10] of atomistic growth models. The exponents which describe this approximate multiscaling behavior are non-universal; their values depend on the way the instability is controlled. The overall picture that emerges from this study is qualitatively similar to that suggested in the analytic work of Ref. [10]. In particular, our work suggests that the multiscaling behavior observed [8] in the 1d Das Sarma-Tamborenea (DT) model is described by the LD equation supplemented by a set of higher-order nonlinear terms with appropriate coefficients.

As noted in Ref. [8], the multiscaling found in simulations of growth models is very similar to the intermittent multifractal behavior observed in fluid turbulence. It is interesting to note in this context that our proposed explanation of multiscaling in growth models is conceptually and formally similar to a recent proposal [15] which suggests that the multiscaling of structure functions in turbulence may be explained in terms of singularities occurring on a dense set of space-time points.

The rest of this paper is organized as follows. In section II, we define the models considered in our study and the various correlation functions measured in our simulations to probe multiscaling behavior. Section III contains a detailed account of the instability we find in discretized growth equations and in an atomistic growth model. In section IV, we describe the results of simulations of models in which the instability is controlled. The behavior found in these simulations is compared and contrasted with the multiscaling behavior observed in previous simulations of the DT and related models. Section V contains a a summary of our findings and a discussion of the implications of our results. A short paper describing the main results of our study has been submitted for publication [16].

\section{MODELS AND DEFINITIONS}

Our work involves detailed numerical studies of two continuum growth equations, namely the LD equation and the KPZ equation. These equations are studied using direct numerical integration. We have also studied by numerical simulations an atomistic version of the LD equation introduced by Kim and Das Sarma [7]. The LD equation we consider has the form

$$
\partial h^{\prime}(\mathbf{r}, t) / \partial t=-\nu \nabla^{4} h^{\prime}+\lambda_{1} \nabla^{2}\left|\nabla h^{\prime}\right|^{2}+\eta(\mathbf{r}, t),
$$

where $h^{\prime}(\mathbf{r}, t)$ represents the "height" variable at the point $\mathbf{r}$ at time $t, \nabla$ and $\nabla^{2}$ represent, respectively, the spatial derivative and Laplacian operators in $d$ dimensions (the dimension of the substrate), and $\eta$ is a Gaussian random noise with correlations

$$
<\eta(\mathbf{r}, t) \eta\left(\mathbf{r}^{\prime}, t^{\prime}\right)>=2 D \delta\left(\mathbf{r}-\mathbf{r}^{\prime}\right) \delta\left(t-t^{\prime}\right) .
$$

The KPZ equation in $(d+1)$ dimensions has the form

$$
\partial h^{\prime}(\mathbf{r}, t) / \partial t=\nu \nabla^{2} h^{\prime}+\lambda_{1}\left|\nabla h^{\prime}\right|^{2}+\eta(\mathbf{r}, t) .
$$

We numerically integrate these two equations using a simple Euler scheme [12,17. To this end, we first define dimensionless variables

$$
\mathbf{x} \equiv \mathbf{r} / r_{0}, \tau \equiv t / t_{0}, h \equiv h^{\prime} / h_{0},
$$

where $r_{0}, t_{0}$ and $h_{0}$ are appropriately chosen units of length, time and height, respectively. We then discretize in space and time by defining the dimensionless discretization scale $\Delta x$ and the integration time step $\Delta \tau$. Using a proper choice of the units $t_{0}$ and $h_{0}$, the equations (11) and (3) can then be represented by the following two update schemes:

$$
h_{i}(\tau+\Delta \tau)-h_{i}(\tau)=\Delta \tau \tilde{\nabla}^{2}\left[-\tilde{\nabla}^{2} h_{i}(\tau)+\lambda\left|\tilde{\nabla} h_{i}(\tau)\right|^{2}\right]+\sqrt{\Delta \tau} \eta_{i}^{\prime}(\tau),
$$

and

$$
h_{i}(\tau+\Delta \tau)-h_{i}(\tau)=\Delta \tau\left[\tilde{\nabla}^{2} h_{i}(\tau)+\lambda\left|\tilde{\nabla} h_{i}(\tau)\right|^{2}\right]+\sqrt{\Delta \tau} \eta_{i}^{\prime}(\tau)
$$


In these equations, $h_{i}(\tau) \equiv h\left(\mathbf{x}_{i}, \tau\right)$ represents the dimensionless height variable at the lattice point $i$ at dimensionless time $\tau, \tilde{\nabla}$ and $\tilde{\nabla}^{2}$ are lattice versions of the derivative and Laplacian operators, and $\eta_{i}^{\prime}(\tau)$ is a random variable with zero average and variance equal to unity. In most of our calculations, we use the following definitions for the lattice derivatives:

$$
\begin{aligned}
\tilde{\nabla}_{j} f_{i} & =0.5\left[f\left(\mathbf{x}_{i}+\mathbf{j} \Delta x\right)-f\left(\mathbf{x}_{i}-\mathbf{j} \Delta x\right)\right], \\
\tilde{\nabla}_{j}^{2} f_{i} & =f\left(\mathbf{x}_{i}+\mathbf{j} \Delta x\right)+f\left(\mathbf{x}_{i}-\mathbf{j} \Delta x\right)-2 f\left(\mathbf{x}_{i}\right),
\end{aligned}
$$

where $\mathbf{j}$ is an unit vector in the $j$ th direction. In some of our calculations, we have also used a more accurate representation [18 of the lattice derivatives involving two neighbors on each side. The dimensionless parameter $\lambda$ appearing in Eqs (5) and (6) has the form

$$
\lambda=\sqrt{2}\left(a_{0} / l_{0}\right)^{(4-d) / 2}, l_{0} \equiv\left(\frac{\nu^{3}}{\lambda_{1}^{2} D}\right)^{1 /(4-d)}
$$

for the LD equation and

$$
\lambda=\sqrt{2}\left(a_{0} / l_{0}\right)^{(2-d) / 2}, l_{0} \equiv\left(\frac{\nu^{3}}{\lambda_{1}^{2} D}\right)^{1 /(2-d)}
$$

for the KPZ equation. In these equations, $a_{0} \equiv r_{0} \Delta x$ is the discretization scale (lattice spacing) and $l_{0}$ is a characteristic length determined by the parameters $\nu, \lambda_{1}$ and $D$ of the original continuum growth equation. Note that in $d$ $=1$, the value of $\lambda$ would vanish in the true continuum limit, $\Delta x \rightarrow 0$, for both LD and KPZ equations . However, one should remember that a short-distance cutoff (perhaps of atomic scale, e.g. the lattice spacing) is present in all physical situations, and it is not legitimate to use a value of $\Delta x$ smaller than this cutoff $a_{m i n}$. Therefore, the smallest value $\left(\lambda_{\min }\right)$ that the coupling constant $\lambda$ can have is $\sqrt{2}\left(a_{\min } / l_{0}\right)^{3 / 2}$ and $\sqrt{2}\left(a_{\min } / l_{0}\right)^{1 / 2}$ for the $1 \mathrm{~d} \mathrm{LD}$ and $\mathrm{KPZ}$ equations, respectively.

We have also studied an atomistic version [7] of the LD equation in which the height variables $\left\{h_{i}\right\}$ are integers. The time evolution of this model is defined by the following deposition rule. First, a site (say $i$ ) is chosen at random. Then the quantity

$$
K_{i}\left(\left\{h_{j}\right\}\right)=-\tilde{\nabla}^{2} h_{i}+\lambda\left|\tilde{\nabla} h_{i}\right|^{2}
$$

is calculated for the site $i$ and all its nearest neighbors. Then, a particle is added to the site that has the smallest value of $K$ among the site $i$ and its nearest neighbors. In the case of a tie for the smallest value, the site $i$ is chosen if it is involved in the tie; otherwise, one of the sites involved in the tie is chosen randomly. Note that this model also involves only one dimensionless parameter, $\lambda$. In this model, "time" is measured by the number of layers deposited. We call this model the KD model [7] below.

The possibility of multiscaling was investigated in our simulations by monitoring different moments of the nearestneighbor height difference and the height difference correlation function. Following Ref. [8], we define

$$
\sigma_{q}(\tau) \equiv<\left(s_{i}(\tau)\right)^{q}>^{1 / q}, s_{i}(t)=\left|h_{i+1}(\tau)-h_{i}(\tau)\right|,
$$

and

$$
G_{q}(l, \tau) \equiv<\left|h_{i+l}(\tau)-h_{i}(\tau)\right|^{q}>^{1 / q},
$$

where we have used the simplified notation $h_{i+l}=h\left(x_{i}+l \Delta x\right)$ for the $1 \mathrm{~d}$ system. In these equations, the average $\langle\ldots\rangle$ represents an average over the site index $i$ and different runs corresponding to different realizations of the random noise. Before saturation (i.e. for $\tau \ll L^{z}$ where $L$ is the size of the system and $z$ is the dynamical exponent), the quantities $\left\{\sigma_{q}(\tau)\right\}$ are expected to show a power-law growth in time $\tau$ in models which exhibit anomalous dynamic scaling:

$$
\sigma_{q}(\tau) \approx \tau^{\alpha_{q} / z}
$$

If the exponent $\alpha_{q}$ depends on the value of $q$, then the model is said to exhibit multiscaling. Thus, whether multiscaling is present or not can be easily tested by monitoring the ratios $\sigma_{q}(\tau) / \sigma_{1}(\tau), q=2,3$.. as functions of time. The height-difference correlation functions $G_{q}$ are expected to behave as

$$
G_{q}(l, \tau) \approx|l|^{\zeta_{q}}, 1 \ll l \ll \xi(\tau) \approx \tau^{1 / z} .
$$

Again, multiscaling, characterized by a dependence of the exponents $\zeta_{q}$ on $q$, can be tested by looking at the $l$ dependence of the ratios $G_{q}(l) / G_{1}(l), q=2,3$ etc. In our work, we consider the first four moments, $q=1,2,3$ and 4. We follow the notation of Ref. [8], in contrast to the notation of Ref. [9], throughout this paper. 


\section{INSTABILITY IN DISCRETE GROWTH EQUATIONS}

In this section, we describe in detail the numerical calculations which lead to the conclusion that a generic instability is present in the discretized growth equations defined in the preceding section. We first studied the behavior of the 1d LD equation, Eq.(5), for small values of the parameter $\lambda(\lambda \leq 2)$. In these runs, the system was started from a perfectly flat state and its time evolution was simulated by integrating the growth equations forward in time. Typical values of the parameters used in the simulation are system size $L=10^{3}$, time step $\Delta \tau=0.01$ and maximum time $\tau_{\max }=10^{4}$. The results were averaged over $10-50$ independent runs. In these runs for small values of $\lambda$, we find good agreement with the predictions of dynamical renormalization group (DRG) calculations [4, 19] and no evidence of multiscaling. In particular, the exponent $\beta$ that describes the growth of the rms interface width $W$ with time is found to have a value $(\simeq 0.34)$ which is close to the DRG result, $\beta=1 / 3$. We also find that the exponents $\left\{\alpha_{q} / z\right\}$ are essentially independent of $q$ and have a value close to zero (in the range $0.06-0.08$ ), possibly indicating a logarithmic growth in time. Typical results obtained for $\lambda=1.0$ are shown in Fig.1. As shown in the inset of this figure, the quantities $\left\{G_{q}(l)\right\}$ also do not show any indication of multiscaling. The exponents $\left\{\zeta_{q}\right\}$ have values in the range 0.8 - 0.9, and are independent of $q$ within error bars. The results obtained from simulations of the KD model for such small values of $\lambda$ are very similar to those described above. Results obtained for $\lambda=0.5$ are shown in Fig.2. The exponents calculated from the time-dependence of $W$ and $\sigma_{q}$ are, respectively, $\beta \simeq 0.345$ and $\alpha_{q} / z \simeq 0.085$ for all $q$. The behavior of the functions $\left\{G_{q}(l)\right\}$, shown in the inset of Fig.2, indicates single-exponent scaling with $\zeta_{q} \simeq 0.9$ for all $q$.

The behavior observed for higher values of $\lambda$ is quite different. In this case, the system exhibits the expected conventional scaling behavior at short times. However, an apparent "singularity", indicated by a rapid growth of the height variable, is found to occur at longer times. It is impossible to follow numerically the evolution of the system beyond the time at which this singularity occurs: attempts to do so lead to "overflow" on the computer. This instability was first observed by $\mathrm{Tu}[12$; ; our results are quite similar to those reported by him. The time at which this instability occurs shows large run-to-run variations, with the average value decreasing with increasing $\lambda$. A similar instability is found in the KD model. Since the height variable in this atomistic model can increase by only one unit at a time, there is no divergence here. The instability in this model shows up as a rapid increase of the interface width which corresponds to a changeover from a power-law growth with an exponent close to $1 / 3$ to a linear growth in time. The results obtained from a simulation of the KD model with $\lambda=1.0$ are shown in Fig.3. The occurrence of an instability near $\tau=100$ is clearly seen in the figure. It is interesting to note that the behavior of $W$ and $\left\{\sigma_{q}\right\}$ before the occurrence of the instability is very similar to that found in simulations for small values of $\lambda$ (see Fig.2). The occurrence of this instability was reported in Ref. [7]. Thus, the observation of these instabilities is not new. Our new results are about the origin of this instability, the apparently "generic" nature of this instability (in the sense that it appears to be present in discretized versions of other commonly studied nonlinear growth equations such as the KPZ equation), and the role it plays in the multiscaling phenomena observed [8 10] in simulations of atomistic growth models.

We carried out a detailed investigation of the origin of this instability in the discretized LD equation and the KD model and found that this instability is caused by the growth of isolated structures, such as pillars and grooves, on a flat interface. Either pillars or grooves are unstable in a particular system; which one is unstable is determined by the sign of $\lambda$. This asymmetry between pillars and grooves results from the fact that the growth equations we consider are not invariant under $h \rightarrow-h$. We find that pillars with heights exceeding a certain "critical" value (which depends on the value of $\lambda$, see below) grow in time in both the LD equation and the KD model with positive $\lambda$. It is easy to show that in the absence of noise $\left(\eta^{\prime}=0\right)$, an isolated pillar of height $h_{0}$ will initially grow in time if $h_{0}>10 / \lambda$. Consider an initial configuration in which all sites except the central one have $h_{i}=0$ and the central site has a height $h_{0}>0$ (a negative value of $h_{0}$ would correspond to a groove at the center). The initial value of time derivative of the height at the central site is easily evaluated from Eq. (5) to be $-6 h_{0}+\lambda h_{0}^{2} / 2$. Similarly, the initial time derivative of the height at one of nearest-neighboring sites of the central one is obtained to be $4 h_{0}-\lambda h_{0}^{2} / 2$. Clearly, the rate at which the difference between the heights at the central site and at one of its nearest-neighboring sites initially changes with time is positive (i.e. the height of the pillar increases initially) if $h_{0}>10 / \lambda$. No analytic method is available for following the evolution of this state for longer times or for taking into account the effects of the stochastic noise $\eta^{\prime}$. We therefore do this numerically and check at regular time intervals whether the nearest-neighbor height difference at the central site (defined as the larger of the two height differences on the two sides) exceeds $h_{0}$ or not. By repeating this procedure a large number of times, we are able to calculate the probability $P(\tau)$ of the nearest-neighbor height difference at the center exceeding the initial value $h_{0}$ at a later time $\tau$. The results of such a study (for $L=100, \lambda=1.0, \Delta \tau=0.01$, 2000 independent runs) are shown in Fig.4. The probability of height increase is found to be very close to zero for small values of $h_{0}$. The growth probability begins to be non-zero as the value of $h_{0}$ exceeds $10 / \lambda$. For values of $h_{0}$ which are slightly higher than $10 / \lambda$, the growth probability is initially close to unity, but it decreases rather quickly 
to zero (see the data for $h_{0}=14$ and 17 in Fig.4), indicating that the height eventually decreases after an initial increase. The rate of the initial growth of the height and the length of the time interval over which the height remains greater than $h_{0}$ increase with $h_{0}$. As $h_{0}$ is increased further, we encounter the instability mentioned above. The height differences near the center grow very rapidly, leading to overflow on the computer. To avoid this problem, we stop the simulation of the time evolution when the maximum value of the nearest-neighbor height difference exceeds a preassigned cutoff value. This cutoff was chosen to be 1000 for the data shown in Fig.4. The results are insensitive to the value of this cutoff as long as it is large. In all runs stopped in this way, the nearest-neighbor height difference at the central site is found to be larger than $h_{0}$ when the run is stopped. These runs are counted as ones in which the nearest-neighbor height difference at the center would remain greater than $h_{0}$ at later times. In fact, the large value of the probability at $\tau=1$ for $h_{0}=20$ (see Fig.4) arises exclusively from such runs. In other words, the height difference at the center becomes smaller than $h_{0}$ within a short time if the height differences do not exceed the cutoff value during the time evolution of the system. This observation and the results of a rigorous analysis [20] of the LD equation without noise suggest that the instability described above is not a true finite-time singularity: the height of the pillar eventually decreases after reaching a large but finite value. Here, we do not address the issue of occurrence of a finite-time singularity in this model because it is virtually impossible to determine numerically whether a true divergence of the height occurs or not. This question is not crucial to our study: as described in section IV, our main results are derived from models in which the growth of the height difference is cut off at a finite value.

As shown in Fig.4, the probability of growth becomes essentially independent of time near $\tau=1$. Fig. 5 shows how the probability at $\tau=1$ depends on the value of $h_{0}$. We show data obtained using three different values, $0.01,0.001$ and 0.0001 , of the time step $\Delta \tau$. The observation that the results obtained for these three very different values of $\Delta \tau$ are nearly identical shows that this instability is not a numerical artifact of not using a sufficiently small value of the time step. From data of this kind, we define a "critical" height $h_{c}$ for which the probability of growth is 0.5 . Our results indicate that the dependence of $h_{c}$ on $\lambda$ is of the form

$$
h_{c}(\lambda) \simeq A / \lambda
$$

with $A \simeq 20.0$ for the LD equation. Our numerical results for the values of $h_{c}$ are shown in Fig.6. The proportionality of $h_{c}$ to $1 / \lambda$ may be understood from a simple dimensional argument. For a configuration in which the height variable is $h_{0}$ at the central site and zero everywhere, the first term on the right-hand side of Eq.(5), which tends to stabilize the system, is proportional to $h_{0}$ at the central site and its nearest-neighbors. The second term on the right-hand side of Eq.(5), which is the one responsible for the instability, is proportional to $\lambda h_{0}^{2}$. It is, therefore, obvious that the value of $h_{0}$ at which the destabilizing term wins over the stabilizing one should be proportional to $1 / \lambda$. The value of the coefficient of proportionality $A$ is nontrivial and has to be determined numerically. We have also carried out similar calculations using a more accurate, five-point definition 18 of the lattice derivatives. We find very similar behavior, with a value of the parameter $A$ which is smaller than 20 . This observation indicates that the behavior described above is not an artifact of using overly simple expressions for the lattice derivatives.

The development of the instability induced in the discretized $1 \mathrm{~d} \mathrm{LD} \mathrm{equation} \mathrm{by} \mathrm{the} \mathrm{presence} \mathrm{of} \mathrm{a} \mathrm{high} \mathrm{pillar} \mathrm{in} \mathrm{the}$ initial state is illustrated in Fig.7 where we show the height profiles at times $\tau=0.05,0.1,0.15$ and 0.17 , obtained by integrating the discretized LD equation $\left(L=100, \lambda=1.0, \Delta \tau=10^{-4}\right)$ from an initial state in which the height is zero everywhere except at the central site where the height is $h_{0}=25$. This value of $h_{0}$ is higher than the critical height $h_{c}$ for the value of $\lambda$ used. As expected, the height of the pillar at the center grows rapidly in time. At the same time, alternate grooves and pillars form on both sides of the initial pillar and these grooves (pillars) become higher (deeper) as time progresses. The formation of these grooves and pillars is a consequence of the conservation law built into the LD equation. In the run depicted in Fig.7, the maximum value of the nearest-neighbor height difference exceeded the cutoff of 1000 at time $\tau=0.2$.

Very similar results are obtained for the atomistic KD model. A little algebra, similar to that described above, shows that in this model, an attempt to deposit a "particle" at the site of a pillar of initial height $h_{0}$ or at one of its nearest-neighboring sites leads to an increase in the height of the pillar if $h_{0}>12 / \lambda$. Our simulations (which are exact because all variables in this model are discrete) show that the height of a pillar continues to grow linearly in time if its initial value is somewhat larger than $12 / \lambda$. The development of the instability in this model is very similar to that shown in Fig.7 for the discretized LD equation.

The instability described above appears to be generic to discretized versions of all commonly studied growth equations containing nonlinear terms. In particular, we have found very similar results for two other systems: the LD equation in $(2+1)$ dimensions and the KPZ equation in $(1+1)$ dimensions. All the qualitative features of the instability found in the $1 \mathrm{~d} \mathrm{LD}$ equation appear to be the present in two dimensions. Pillars of initial height $h_{0}$ become unstable in the 2d LD equation with positive $\lambda$ if $h_{0}>h_{c}(\lambda)$. The dependence of $h_{c}$ on $\lambda$ is well-described by $h_{c}(\lambda) \simeq A / \lambda$ with $A \simeq 31$ (see Fig.6).

The instability in the discretized KPZ equation, Eq.(6), in one dimension with $\lambda>0$ is associated with grooves, not pillars. We have studied the $1 \mathrm{~d} \mathrm{KPZ} \mathrm{equation} \mathrm{with} \mathrm{and} \mathrm{without} \mathrm{noise} \mathrm{and} \mathrm{found} \mathrm{the} \mathrm{instability} \mathrm{to} \mathrm{be} \mathrm{very} \mathrm{similar}$ 
in the two cases. The critical value of $h_{0}$ (the depth of an isolated groove) in the KPZ equation is determined using a procedure similar to the one described above for the LD equation. The instability criterion we use for the KPZ equation is slightly different from the one described above. We define the probability of occurrence of an instability as the ratio between the number of runs in which the maximum nearest-neighbor height difference exceeds a preassigned cutoff value (taken to be 1000 in our simulations) and the total number of runs. As noted above, this instability criterion coincides with the criterion of the value of the nearest-neighbor height difference at the central site exceeding $h_{0}$ in the LD equation. This is not so in the $1 \mathrm{~d} \mathrm{KPZ}$ equation. In some of the runs, we find that the value of the nearest-neighbor height difference at the central site is smaller than $h_{0}$ when the maximum value of the nearestneighbor height difference reaches the cutoff. Evidently, the presence of a deep groove in the initial state induces the formation of large height differences at points which do not always coincide with the initial location of the groove. The development of the instability in the 1d discretized KPZ equation is shown in Fig.8. The growth profiles shown for times $\tau=0.1,0.3$ and 0.5 are obtained for a $L=100$ system with $\lambda=1.0$, using an integration time step $\Delta \tau=10^{-4}$. The initial state is one in which the height is zero everywhere except at the central site where there is a groove of depth 30. As can be seen in Fig.8, the groove at the center becomes deeper initially, but subsequently develops into a "mound" with large values of the nearest-neighbor height difference occurring at many points near the center. This is the reason why the maximum value of the nearest-neighbor height difference does not always occur at the central site in this system. A comparison of Fig.8 with Fig.7 clearly illustrates the important effects of a conservation law (which is present in the LD equation, but absent in the KPZ equation) on the growth kinetics. The value of the maximum nearest-neighbor height difference was found to exceed the cutoff of 1000 at $\tau=2.7$ in the run for which the results are shown in Fig.8.

Typical results for the probability of occurrence of an instability in the $1 \mathrm{~d}$ KPZ equation $(\lambda=1.0, L=100, \Delta \tau$ $=0.01,2000$ runs) are shown in Fig.9 for three different values of $h_{0}$. As before, we define $h_{c}$ to be the value of $h_{0}$ at which the long-time value of the probability of instability reaches 0.5 . The dependence of $h_{c}$ calculated in this way on the value of $\lambda$ is shown in Fig.6. As expected, we find $h_{c}(\lambda) \simeq A / \lambda$ with $A \simeq 25.0$. The values of $h_{c}$ shown in Fig.6 were obtained from numerical integrations using a time step $\Delta \tau=0.01$. We have repeated these calculations using smaller values of $\Delta \tau$. The observed dependence of the calculated value of $h_{c}$ on $\Delta \tau$ is more pronounced than that shown in Fig.5 for the 1d LD equation. However, any reasonable extrapolation of the results obtained for different values of $\Delta \tau$ to the $\Delta \tau \rightarrow 0$ limit yields results for $h_{c}$ which are not significantly different from those shown in Fig.6. We have also checked directly the occurrence of the instability in the 1d discretized KPZ equation without noise for initial conditions containing a deep groove for values of $\Delta \tau$ down to $10^{-7}$.

Our conclusion about the existence of an instability in the discretized version of the noiseless $1 \mathrm{~d}$ KPZ equation may appear surprising in view of the well-known fact that the continuum KPZ equation without noise in one dimension does not have any instability. The continuum equation can be mapped to a simple linear diffusion equation by a Cole-Hopf transformation and solved exactly. For any bounded initial condition, the asymptotic solution is one in which the height variable is constant. The absence of any instability in the continuum equation, however, does not necessarily imply that the discretized version, Eq.(6), should also be stable for any initial condition. This is because the application of a Cole-Hopf transformation to the discretized version (cf. Eq.(6)) of the KPZ equation does not reduce it to a discretized version of the linear diffusion equation. The reason for this difference is simple: the algebra of derivatives does not apply to difference operators if the nearest-neighbor height differences are not vanishingly small. For this reason, the exact results available for the continuum equation do not in any way rule out the possibility of occurrence of an instability in the discretized equation for initial conditions with large nearest-neighbor height differences. Conclusions very similar to ours about the occurrence of an instability in the 1d discretized noiseless KPZ equation have recently been obtained independently by Newman and Bray [21].

It is interesting to note that instabilities in numerical integrations of the discretized KPZ equation in one and higher dimensions were noted in previous studies [13]. These studies, however, attributed the observed instability to "numerical artifacts", with the implicit assumption that the instability would disappear if a sufficiently small value of the integration time step $\Delta \tau$ were used. Our work shows, for the first time, that the instability found in these studies is an intrinsic property of the discretized equation which can not be eliminated simply by using a sufficiently small value of $\Delta \tau$. The observation of an instability in the discrete version of the noiseless KPZ equation in one dimension brings out another important point which, to our knowledge, has not been noted in the existing literature, namely, the behavior of discretized versions of nonlinear growth equations may, under certain circumstances, be very different from the behavior of their continuum counterparts. These new observations have several important implications in the study of growth equations. In particular, one important and inevitable implication is that the discrete version of a nonlinear continuum growth equation may, in principle, belong to a universality class which is different from the universality class of the continuum equation. A full discussion of these implications is provided in section $\mathrm{V}$.

So far, we have considered the time evolution of the discretized growth equations from an initial state in which a pillar or groove is present. A question of obvious importance is whether such structures are spontaneously generated during the evolution of the system from a flat initial state. The answer to this question is crucially related to the 
nature of dynamic scaling exhibited by the model under consideration. In systems which exhibit normal scaling, the nearest-neighbor height difference is not expected to grow indefinitely in time; it should saturate quickly after an initial growth. Such a system would spontaneously exhibit the instability discussed above only if the value at which the maximum nearest-neighbor height difference $s_{\max }$ saturates is higher than (or at least, close to) the critical value, $h_{c}$, defined above. Since $h_{c}$ decreases while the saturation value of $s_{\max }$ generally increases with $\lambda$, we can define a non-zero "critical" value, $\lambda_{c}$, of $\lambda$ at which these two quantities become equal. According to the discussion above, systems with values of $\lambda$ substantially smaller than $\lambda_{c}$ are not expected to show the instability during their time evolution from a flat state. In contrast, since nearest-neighbor height differences are expected to continue growing in time in models which exhibit anomalous scaling, such systems should always show the instability at sufficiently long times, provided the system size is large enough to prevent saturation before the onset of the instability. In other words, $\lambda_{c}$ is expected to be zero for models with anomalous scaling. This conclusion is different from that of Tu [12] who interpreted his numerical results for the discretized 1d LD equation as evidence for the existence of a non-zero $\lambda_{c}$ in this system.

Our numerical results fully support these general conclusions. In Fig.10, we show the time-dependence of $s_{\max }$, the maximum value of the nearest-neighbor height difference averaged over a large number of runs starting from a flat state. The system parameters are $L=1000, \Delta \tau=0.01, \lambda=4.0$ for the $1 \mathrm{~d}$ LD equation; system size $=200 \times 200$, $\Delta \tau=0.01, \lambda=5.0$ for the $2 \mathrm{~d} \mathrm{LD}$ equation; and $L=10^{4}, \Delta \tau=0.01, \lambda=5.0$ for the $1 \mathrm{~d} \mathrm{KPZ}$ equation. A larger value of $L$ is needed for avoiding saturation in the KPZ equation because the value of $z$ for this model is smaller. The quantity $s_{\max }$ is defined in the following way for the $2 \mathrm{~d}$ system:

$$
s_{\max } \equiv \max \left\{s_{i}\right\} ; s_{i} \equiv\left[\left(h\left(\mathbf{x}_{i}+\mathbf{i} \Delta x\right)-h\left(\mathbf{x}_{i}\right)\right)^{2}+\left(h\left(\mathbf{x}_{i}+\mathbf{j} \Delta x\right)-h\left(\mathbf{x}_{i}\right)\right)^{2}\right]^{1 / 2} .
$$

The data shown were averaged over 200, 30 and 200 runs for the $(1+1) \mathrm{LD},(2+1) \mathrm{LD}$ and (1+1) KPZ equations, respectively. As expected, $s_{\max }$ saturates quickly for the last two models which are expected to exhibit normal scaling behavior. In contrast, $s_{\max }$ continues to grow in time in the $1 \mathrm{~d}$ LD equation which is expected to show anomalous scaling. The growth of $s_{\max }$ in time in this model is well-described by a logarithmic form

$$
s_{\max }(\tau) \approx a+b \ln \tau,
$$

where the parameter $b$ is numerically found to be proportional to $\lambda$. This logarithmic dependence of $s_{\max }$ on $\tau$ is consistent with predictions of dynamical renormalization group calculations [4, 19].

Fig.11 shows our numerical results for $\tau_{\text {ins }}$, the time at which the instability occurs when the system evolves from a flat initial state, for $1 \mathrm{~d}$ LD and KPZ equations. We operationally define $\tau_{\text {ins }}$ as the time at which $s_{\text {max }}$ reaches a cutoff value which is set at 1000 . The instability time shows very large run-to-run variations, and we find it more appropriate to average $\ln \tau_{\text {ins }}$, rather than $\tau_{\text {ins }}$ itself, over different runs. So, the data shown in Fig.11 actually correspond to $\exp \left(<\ln \tau_{\text {ins }}>\right)$. The data for the $\mathrm{LD}$ equation were obtained for $L=1000, \Delta \tau=0.01$, and averaged over 150, 200, 500, 1000 and 1000 independent runs for $\lambda=3,4,5,6$ and 7, respectively. From the results of Eqs (16) and (18), and the fact that the coefficient $b$ of Eq. (18) is proportional to $\lambda$, it is easy to show that the dependence of $\tau_{\text {ins }}$ on $\lambda$ in this model should given by $\tau_{\text {ins }} \approx e^{B / \lambda^{2}}$. As shown in Fig.11, this form does provide a good description of the numerical data for small values of $\lambda$. The fact that the values of $\tau_{\text {ins }}$ for $\lambda=6$ and 7 are lower than those predicted by the fit to the data for smaller $\lambda$ may be understood by noting that the initial growth of $s_{\max }$ with time is faster that that described by Eq.(18).

The data shown for the $1 \mathrm{~d} \mathrm{KPZ}$ equation were obtained for $L=10^{4}, \Delta \tau=0.01$, and averaged over 200 runs. In 100 runs of length $10^{4}$ units, we did not find any occurrence of instability for $\lambda=4$. From the observation that the distribution of $\tau_{i n s}$ has a long tail that extends to values much smaller than the average, we can derive a conservative lower limit of $10^{6}$ units for the average instability time for $\lambda=4$. This is indicated by the arrow in Fig.11. These results strongly suggest that the value of $\lambda_{c}$ lies between 4 and 5 in this system. Consequently, the instability in this system may be avoided by choosing a small discretization scale $\Delta x$, one which would make the value of $\lambda$ smaller than $\lambda_{c}$. However, as noted before, the value of $\lambda$ in a real system can not be made arbitrarily small, and the instability can not be avoided if the "bare" parameters are such that $\lambda_{\min }>\lambda_{c}$.

It should be mentioned that the above discussion about the possibility of occurrence of an instability in a system started from a flat initial state is qualitative because it is based on a criterion that involves the average value of the nearest-neighbor height difference $s_{\max }$. Our simulations show that the value of $s_{\max }$ at a particular time $\tau$ exhibits large run-to-run variations. The distribution of $s_{\max }$ shows a long tail extending to values substantially higher than the average value. This variation in the value of $s_{\max }$ is the main reason for the large fluctuations in the calculated value of $\tau_{\text {ins }}$. Since an instability is expected to occur whenever the value of $s$ exceeds $h_{c}$, a system with a value of $\lambda$ which is lower than the $\lambda_{c}$ defined above in terms of the average value of $s_{\max }$ would exhibit the instability if a large value of $s$ lying in the tail of the distribution happens to be generated during the time evolution. Since the tail of the 
distribution is more likely to be sampled if the system size is large and if the simulation is continued for long times, the probability of occurrence of an instability in a system with $\lambda<\lambda_{c}$ would increase with system size and simulation time. These considerations show that a precise definition of $\lambda_{c}$ is problematic. All we can say with certainty is that for sample sizes and simulation times used in typical numerical integrations of growth equations starting from a perfectly flat state, an instability would be very unlikely in a system exhibiting conventional (rather than anomalous) dynamic scaling if the value of $\lambda$ is significantly smaller than the critical value $\lambda_{c}$ defined above. As discussed above, the results of our numerical investigation of the time of instability are quite consistent with this prediction.

We also note that the distinction we make between models with $\lambda_{c}=0$ (e.g. the $1 \mathrm{~d}$ LD equation) and those with $\lambda_{c}>0$ (e.g. the KPZ equation and the $2 \mathrm{~d} \mathrm{LD}$ equation) is appropriate only when one considers the evolution of the system from a flat initial state. Models with non-zero values of $\lambda_{c}$ would show the instability (even if the value of $\lambda$ is smaller than $\lambda_{c}$ ) if the initial state has a sufficiently high pillar or a sufficiently deep groove (i.e. if $h_{0}>h_{c}(\lambda)$, which is finite for any non-zero value of $\lambda$ ). If the LD and KPZ equations are indeed the appropriate continuum descriptions of epitaxial growth, as is currently believed [1]2] to be the case, then our finding of this generic instability for growth on a substrate with a high pillar/groove may have important implications for real growth on patterned substrates, which is a subject of considerable current interest in materials science.

\section{CONTROLLED INSTABILITY AND MULTISCALING}

In this section, we describe in detail our numerical investigation of the connection between the instability described in the preceding section and multiscaling behavior. The existence of such a connection was suggested by the following observation made in our numerical studies of the discretized LD equation and the KD model in one dimension. We found evidence for multiscaling (as indicated by the observed time-dependence of the ratios $\sigma_{q} / \sigma_{1}$ ) during a short interval of time immediately preceding the instability. During this time interval, the interface width shows the expected scaling behavior, but the functions $\left\{\sigma_{q}\right\}$ appear to scale with different powers, the growth in time being faster for larger values of $q$. This observation suggests that multiscaling behavior may be closely related to this instability. We, therefore, carried out a detailed investigation of this aspect.

It is easy to see that the instability described above would, in general, lead to deviations from single-exponent scaling for the quantities $\left\{\sigma_{q}\right\}$. When the instability sets in, the value of the nearest-neighbor height difference $s$ at the point of instability becomes large and it grows rapidly in time. Since higher moments of $s$ (i.e $\sigma_{q}$ for large $q$ ) are more sensitive to such large values of $s$, the growth of $\sigma_{q}$ in time would be faster for larger values of $q$. The instability would also produce a long tail extending to large values in the distribution of $s$, leading to departures from single-exponent scaling for the correlation functions $\left\{G_{q}\right\}$. As mentioned above, we do find approximate multiscaling in our simulations near the onset of the instability. Typical data, obtained for the LD equation $\left(L=10^{3}, \lambda=4.0\right.$, 100 runs) in one dimension, are shown in Fig.12. The growth of the ratio $\sigma_{q} / \sigma_{1}$ with time is clearly seen, especially for large values of $q$. We have shown data only upto $\tau=100$ because the instability is encountered at longer times and the time evolution of the system can not be followed beyond the instability. Thus, the time interval over which multiscaling is observed in the systems considered so far is very short. This is because the instability in these systems is very "strong" in the following sense. In the discretized growth equations, the time evolution of the system can not be followed numerically beyond the instability time because the height variables become too large. In the atomistic KD model, the height variables increase so fast after the onset of the instability that global variables such as the width of the interface begin to show deviations from scaling (see, for example, Fig.3). In order to explain the numerical results obtained in Ref. [8 10], it is necessary to have a situation in which the global variables scale in a normal way, whereas the quantities $\left\{\sigma_{q}\right\}$ and $\left\{G_{q}\right\}$ show anomalous multiscaling. The discussion above suggests that such a situation may be realized if the instability is "controlled" in some way. We have considered two different classes of models with controlled instability. In models of the first class, the instability is controlled by the introduction of terms with higher powers of the gradient of the height variable with appropriate coefficients. The second class consists of atomistic models in which the instability is controlled by modifying the deposition rule in a way that restricts the development of large values of the nearest-neighbor height difference. These models and the results obtained from numerical studies of their behavior are described below. We emphasize that there is no unique way to control the growth instability, and, in principle, there must be infinitely many different ways to do it. We have tried several simple techniques of controlling the instability using minimal number of parameters, as described in the next two subsections. In general, for a given atomistic growth model (e.g. the DT model), the instability may be controlled in an unknown and necessarily non-universal manner. 


\section{A. Controlled Instability Models with Higher Powers of the Gradient}

The models we consider in this section are obtained by replacing the $\left|\tilde{\nabla} h_{i}\right|^{2}$ term appearing in Eqs.(5) and (11) by $f\left(\left|\tilde{\nabla} h_{i}\right|^{2}\right)$, where $f$ is the following nonlinear function:

$$
f(x) \equiv\left(1-e^{-c x}\right) / c .
$$

In the equation above, $c$ is an adjustable parameter. Note that this replacement corresponds to the introduction of an infinite number of higher-order nonlinear terms of the form $\left|\tilde{\nabla} h_{i}\right|^{2 n}$ with specific coefficients which depend on the value of $c$. In the following, we call this modified version of the discretized LD equation the controlled Lai-Das Sarma (CLD) model and the modified version of the KD model is referred to as the controlled Kim-Das Sarma (CKD) model. The function $f(x)$ behaves as $x$ for $x \ll 1 / c$ and approaches a constant value, $1 / c$, in the limit $x \gg 1 / c$. It is easy to show that the growth instability of isolated pillars found in the original LD equation and the KD model is completely suppressed if the value of $c$ is higher than a critical value which depends on the value of $\lambda$. For values of $c$ smaller than this critical value, the instability occurs for an isolated pillar if its height lies within a range $h_{\min }(\lambda, c)<h_{0}<h_{\max }(\lambda, c)$. This is shown in Fig.13 where we have plotted the quantity

$$
\Delta\left(h_{0}\right) \equiv \frac{d}{d \tau}\left[h_{n}-h_{n-1}\right]
$$

for the noiseless CLD model for a state with a pillar of height $h_{0}$ at the $n$-th site (i.e. for a configuration with $h_{i}=h_{0}$ for $i=n$ and $h_{i}=0$ for all other $i$ ). The results shown are for $\lambda=4.0$ and three different values of the control parameter $c$. A positive value of $\Delta$ implies that the pillar initially grows in time. As discussed in the preceding section, an initial growth of the height of a pillar is a necessary (but not sufficient) condition for the occurrence of the instability. It is clear from the figure that there is no instability for $c=0.3$, while the model would exhibit an instability for values of $h_{0}$ lying within a limited range for $c=0.05$ and 0.02 . The range of $h_{0}$ values for which the instability is expected to occur clearly becomes wider as the value of $c$ is decreased. Simulations carried out for the CLD model with noise show an essentially similar behavior. The CKD model also behaves in a very similar way. Fig.14 shows the probability of growth of an isolated pillar of initial height $h_{0}$ in the CKD model with $\lambda=2.0$ and $c=0.02$. The growth probability is defined as before as the fraction of runs in which the nearest-neighbor height difference at the site of the pillar exceeds $h_{0}$. The growth probability is found to be close to zero at all times for $h_{0}$ $=5$ and $h_{0}=45$, indicating that these values are, respectively, lower than $h_{\min }$ and higher than $h_{\max }$. For $h_{0}=25$, on the other hand, the growth probability increases quickly to a value close to unity and then falls off at long times. Thus, the instability for large value of $h_{0}$ is controlled in the new models by the introduction of an infinite series of higher powers of the derivative with appropriate coefficients. This is physically reasonable because terms involving higher powers of the derivative are expected to come into the picture when the derivative itself becomes large.

We have studied numerically the behavior of both CLD and CKD models for different values of $\lambda$ and $c$. The numerical divergence found in the original discretized LD equation disappears completely for any non-zero value of $c$. This result confirms that the instability we found in the original LD equation is a genuine one, not an artifact of our numerics. The behavior we find in these two models are qualitatively very similar. For values of $c$ which are so large that the instability is completely absent, we find conventional scaling with exponents close to the expected values. For very small values of $c$, the instability is very "strong" and we find deviations from scaling for global quantities such as the interface width. Typical results are shown in Fig. 15 where we have shown the time dependence of the interface width $W$ for the CLD model $\left(\mathrm{L}=10^{3}, \lambda=4.0\right)$ with two values of $c$ (10 runs for $c=0.02$ and 40 runs for $\left.c=0.05\right)$, and for the CKD model $\left(L=10^{3}, \lambda=2.0\right)$, also with two different values of $c(c=0.02,200$ runs and $c=0.005$, 10 runs). In both models, the results for the larger value of $c$ show the expected power-law scaling in time, whereas the data for the smaller value of $c$ exhibit a "strong" instability at which the scaling behavior of $W$ breaks down. It is interesting to note that the growth of $W$ before the occurrence of the break in the curves for the smaller values of $c$ is almost indistinguishable from that seen for the larger values of $c$ for which the power-law growth continues till long times. Thus, the scaling behavior of $W$ even in the "strongly" unstable situation mimics the ordinary power-law growth upto the instability onset time which may be very long, depending on the values of the parameters in the model. As noted in section III above, similar results were obtained for the original (uncontrolled) models also. The plots in Fig.15 show explicitly the similarity between the behavior of the two models. We describe below results obtained for the atomistic CKD model because simulations of this model are easier, so that better statistics can be obtained. Very similar results, but with poorer statistics, were obtained for the CLD model.

Interesting behavior is found in simulations of the CKD model with intermediate values of $c$ for which the instability occurs for a limited range of values of $h_{0}$. For such values of $c$, the instability is expected to be operative over a limited time interval. At very early times, the values of $s$ are small and no instability occurs. As time progresses, 
the instability sets in when the value of $s_{\max }$ crosses $h_{\min }$. The value of $s$ at the point of instability grows rapidly in time until the growth is cutoff at $h_{\max }$. At subsequent times, the instability occurs at more and more points in the system. The number of points at which a fresh instability can occur decreases in this process. Also, effects of the instability become less pronounced at long times because the typical value of $s$, which increases with time even if there is no instability, becomes comparable to $h_{\max }$ at sufficiently long times. So, the instability is expected to become ineffective at long times. If multiscaling arises due to the instability, then one expects to see multiscaling only during the finite time interval over which the instability is active. This is precisely the behavior we find in the simulations. In Figs.16 and 17, we show a representative set of simulation results obtained for $L=1000, \lambda=2.0$, and $c=0.02$, averaged over 2000 runs. For these values of $\lambda$ and $c, h_{\min } \simeq 6.0$ and $h_{\max } \simeq 34.0$. As shown in Fig.16, the rms interface width $W$ shows excellent scaling with an exponent close to $1 / 3$. The quantities $\left\{\sigma_{q}\right\}$, however, show clear evidence of multiscaling during the time interval between $\tau \approx 5$ and $\tau \approx 1000$. Power-law fits to the data over this time interval yield the following values for the effective exponents: $\alpha_{1} / z=0.14 \pm 0.02, \alpha_{2} / z=0.17 \pm 0.02, \alpha_{3} / z$ $=0.22 \pm 0.02, \alpha_{4} / z=0.26 \pm 0.03$. These exponent values are similar to those found in Ref. [8] for the $1 \mathrm{~d}$ DT model. As shown in the inset of Fig.16 where we have plotted the time-dependence of the ratios $\sigma_{q} / \sigma_{1}$ for $q=2,3$ and 4 , the multiscaling is not present at very early times and also at times longer than about 1000. By monitoring the time development of $s_{\max }$, we find that $\tau \approx 1000$ is precisely the time at which the instability begins to level off. We have carried out much longer runs for smaller samples and found that multiscaling of $\left\{\sigma_{q}\right\}$ is absent for all $\tau>1000$.

In Fig.17, we have plotted the correlation functions $\left\{G_{q}\right\}$ for the same system at time $\tau=1000$. Multiscaling is clearly seen, with the following exponent values calculated from power-law fits to the data for $2 \leq l \leq 10: \zeta_{1}=0.74$ $\pm 0.03, \zeta_{2}=0.66 \pm 0.03, \zeta_{3}=0.58 \pm 0.03$, and $\zeta_{4}=0.50 \pm 0.03$. These exponent values are also similar to those found in Ref. [8] for the 1d DT model. The multiscaling behavior for $l \leq 20$ is clearly illustrated in the inset where we have plotted the ratios $G_{q}(l) / G_{1}(l)$ for $q=2,3$ and 4 as functions of $l$.

We have also investigated the dependence of $W$ on the sample-size $L$ in this model for small values of $L$. The time-dependence of $W$ in a sample of length $L$ is expected to be described by the finite-size scaling equation

$$
W(\tau, L)=\tau^{\beta} f\left(L \tau^{-1 / z}\right) .
$$

The scaling function $f(x)$ in Eq.(21) goes to a constant as $x \rightarrow \infty\left(\tau \ll L^{z}\right)$, so that the interface width grows as $\tau^{\beta}$ at early times. From the data shown in Fig.16, we estimate the value of $\beta$ to be close to 0.35. The scaling function behaves as $f(x) \approx x^{\beta z}$ in the $x \rightarrow 0$ limit, so that at times long compared to $L^{z}$, the interface width becomes timeindependent and proportional to $L^{\alpha}$ with $\alpha=\beta z$. The value of the exponent $\alpha$ is estimated to be close to 1.25 form the observed sample-size dependence of the interface width at saturation. These exponent values correspond to $z \simeq$ 3.5. The scaling equation (21) can also be written as

$$
W(\tau, L) L^{\alpha}=g\left(\tau L^{-\alpha / \beta}\right)
$$

so that for a proper choice of the values of the exponents $\alpha$ and $\beta$, the data for different $L$ and $\tau$ should collapse to a single scaling curve when $W L^{\alpha}$ is plotted against $\tau L^{-\alpha / \beta}$. A scaling collapse of the data obtained for three different values $(20,40$ and 80$)$ of $L$ for the CKD model with $\lambda=2.0$ and $c=0.02$ is shown in Fig.18. The number of independent runs used in generating the data shown is 2000 for $L=20$ and 1000 for $L=40$ and 80 . The exponent values used in the scaling plot are $\alpha=1.25$ and $\beta=0.355$. Thus, it is clear that the CKD model with these parameters exhibits the expected scaling behavior for the global quantity $W$. These exponent values, when combined with the values of the exponents $\alpha_{q} / z$ and $\zeta_{q}$ quoted above, satisfy the expected relation $\alpha_{q}+\zeta_{q}=\alpha$ within error bars, although there appear to be systematic deviations from this relation for large $q$. Very similar results were obtained in Ref. [8] for the DT model.

We have also calculated the distribution of the nearest-neighbor height difference $s$ at long times. Results for the CKD model with $\lambda=2.0$ and $c=0.02$ are shown in Fig.19. We show the distribution $P(s)$ for two different cases: for a system with $L=10^{3}$ (averaged over 2000 runs) at time $\tau=1000$ (the time at which multiscaling in $\left\{\sigma_{q}\right\}$ tapers off, see Fig.16), and for a system with $L=80$ (1000 runs) averaging over the time interval $5 \times 10^{4} \leq \tau \leq 5 \times 10^{5}$ in the saturation regime. In both cases, the distribution of $s$ is found to be strongly non-Gaussian with a long tail extending to large values of $s$. As shown in the figure, a power-law form, $P(s) \propto s^{-\eta}$, provides an excellent fit to the data over more than four decades. The best-fit value of the exponent $\eta$ of the power law is found to be 3.2 for the $L$ $=1000$ system and 2.5 for the $L=80$ system. This power-law behavior is different from the result obtained [8] for the 1d DT model in which $P(s)$ appears to show a stretched exponential behavior. As shown in the inset of Fig.19, a stretched exponential form with a stretching exponent $\approx 0.6$ provides a good fit to our $L=80$ data for small values of $s$, but fails at larger values.

These results clearly show that multiscaling behavior very similar to that observed in Ref [8 [10] can be generated by a controlled instability of the kind described above. It should, however, be noted that the multiscaling we find is transient in the sense that it occurs only over a limited range of time. A careful look at the data of Ref [8 10] 
suggests that the same is true for the atomistic models studied in these papers. The approximate multiscaling we find is non-universal: the effective exponents $\zeta_{q}$ and $\alpha_{q}$ extracted from our numerical data seem to depend on the way in which the instability is controlled. Similar non-universality is also found in the atomistic models studied in Ref 810$]$.

The similarity between the CKD model with appropriate choice of the parameters and the DT model is also illustrated by a comparison between the growth profiles in the two models. Since both these models are atomistic in nature, it makes sense to compare the growth profiles obtained at the same value of the discrete time measured in units of number of layers deposited. In Fig.20 and Fig.21, we show typical growth profiles in, respectively, the 1d DT model and the 1d CKD model with $\lambda=2.0$ and $c=0.02$. Both profiles are obtained for samples with $L=$ 1000 after the deposition of $10^{4}$ layers. The average value has been subtracted off from the heights plotted in these Figures. The similarity between these two profiles becomes evident when one is inverted relative to the other i.e. if the transformation $h \rightarrow-h$ is made in one of the profiles. This transformation is equivalent to changing the sign of $\lambda$ in the CKD model. Thus, the DT model appears to be similar to the CKD model (and also to the CLD model) with a negative value of $\lambda$. The asymmetry between the peaks and troughs of the profile is evident in both Fig.20 and Fig.21. In the 1d DT model (Fig.20), the peaks of the profile are generally rounded and the troughs tend to be very sharp and "spiky". The profile also wanders a longer distance from the baseline (average height) in the negative direction. Both these features are reversed in the profile obtained in the 1d CKD model (Fig.21). The reason for this "inversion" is quite simple. The profile obtained in the CKD model with positive $\lambda$ exhibits sharp peaks and wanders a longer distance on the positive side because pillars are unstable in this model. The situation is reversed in the DT model because, as shown below, grooves have a finite probability of getting deeper in this model. This difference can be eliminated simply by changing the sign of $\lambda$ in the CKD model.

\section{B. Controlled Instability Models with Modified Deposition Rule}

We have constructed and studied by simulations a modified version of the atomistic KD model in which the deposition rule is changed in order to control the growth of nearest-neighbor height differences. This study was motivated by the following observation. In order to explore further the connection between controlled instability and multiscaling, we studied by simulations the evolution of isolated pillars and grooves in the $1 \mathrm{~d}$ DT model. We start with a configuration which is flat everywhere except at the central point where there is a pillar of height $h_{0}$ or a groove of depth $h_{0}$. We then simulate the time evolution of this state and measure the probability that the absolute value of the nearest-neighbor height difference at the central site exceeds $h_{0}$ at time $\tau$. We find that the probability of a pillar becoming higher is strictly zero whereas grooves have a non-zero probability of becoming deeper. Thus, the asymmetry between grooves and pillars found in the models described above is present in the DT model also. It is not difficult to explain the origin of this asymmetry. Consider a configuration in which $h_{i}$ is zero everywhere except at the site $n$ where $h_{n}=h_{0}$. For a positive $h_{0}$ (a pillar at site $n$ ), a particle deposited at site $n$ diffuses to one of its nearest neighbor sites because the number of bonds at site $n$ is one whereas the number of bonds at the sites $n+1$ and $n-1$ is two. Particles deposited at sites $n+1$ and $n-1$ do not diffuse because each of these two sites have two bonds. Thus, there is no deposition sequence which can increase the height differences $\left|h_{n}-h_{n-1}\right|$ and $\left|h_{n}-h_{n+1}\right|$. In contrast, for a negative $h_{0}$ (a groove at site $n$ ), there are certain deposition sequences which increase these height differences. Consider, for example, the sequence in which a particle is first deposited at the site $n+2$ and then another particle is deposited at the site $n+1$. The particle deposited at $n+2$ stays there because although this site has only one bond, the nearest neighbors of this site also have only one bond each. The particle deposited subsequently at $n+1$ also stays at this site because it now has two bonds. The difference between the heights at sites $n$ and $n+1$ increases in this process. Therefore, the probability of a groove becoming deeper in the course of time should be non-zero in this model. This simple picture also implies that this probability should not depend strongly on $h_{0}$, the initial depth of the groove, as long as $h_{0}$ is not very small. We believe that the non-zero probability of grooves becoming deeper is the basic reason for the occurrence of large values of the nearest-neighbor height difference (which lead to multiscaling) in the 1d DT model. This belief is supported by examinations of the height profiles generated in simulations of the 1d DT model which show that large values of the nearest-neighbor height difference almost always correspond to deep grooves in this system (see, for example, Fig.20).

The results of our simulations on the $1 \mathrm{~d}$ DT model, obtained by averaging over $10^{5}$ runs on a $L=32$ sample, are shown in Fig.22. The data shown were obtained for $h_{0}=100$. The same behavior is found for all values of $h_{0}>10$. These results are consistent with the simple picture described above. The probability of increase of the depth of a groove is found to increase initially with time, reach a maximum near $\tau=1$ and decay slowly to zero at longer times. The shape of the probability vs. time curves in this model is qualitatively similar to that of the curve shown in Fig. 14 for $h_{0}=25$ in the CKD model. There are, however, large quantitative differences between the two curves. Clearly, the 
probability of growth of the nearest-neighbor height difference is substantially smaller and decays faster in time in the DT model. We have also investigated the time evolution of wider grooves in the 1d DT model. A groove of depth $h_{0}$ and width $w$ corresponds to an initial configuration in which the height is $-h_{0}$ at the sites $n, n+1, \cdots, n+w-1$, and zero everywhere else. All values of $n$ are equivalent because periodic boundary conditions are used. From simulations of the time evolution of initial configurations with different values of $w$ and $h_{0}$, we calculate the probability that the difference between the height at the central site of the groove and the average height outside the groove is greater than $h_{0}$ at time $\tau$. As shown in the inset of Fig.22, where we have plotted the results for $w=3$ and $h_{0}=30$ and 90 (these results were obtained by averaging over 1000 runs on samples with $L=128$ ), this probability is quite high and it decays rather slowly with time. The probability of a groove getting deeper is found to increase with increasing $w$ and $h_{0}$. The decay of this probability in time becomes slower as the values of $w$ and/or $h_{0}$ are increased. Detailed examination of the configurations generated in the simulations shows that the difference between the height at the center of the groove and the average height outside the groove does not increase much beyond $h_{0}$, but remains slightly higher than $h_{0}$ with a high probability over a relatively long period of time which increases with $w$ and $h_{0}$. This behavior may be understood in the following way. Consider a groove with $w=3$ centered at the site $n$. Each of the nearest neighbors of the central site $n$ has two bonds in the initial state. Therefore, particles dropped at one of these two neighbors of the central site stay at that site, and the central site does not get any particle from its nearest neighbors during the initial time evolution of the system. There is, however, a finite probability for a particle dropped at the central site to move to one of the neighboring sites. This would happen, for example, if the heights at the sites $n, n+1$ and $n-1$ are equal. So, the rate at which the height at the central site grows initially is slightly lower than the rate of growth of the average height (which, by definition, is one layer per unit time in this model). These considerations do not apply at long times when the groove begins to fill up. For this reason, the probability of the groove getting deeper begins to decrease at long times, making this "unstable" behavior a long-lasting transient. The slow decay of this transient behavior for large values of $w$ and $h_{0}$ may have important implications for real growth on patterned substrates.

An important difference between the results obtained for the DT and CKD models is that the growth probability is close to zero for $h_{0}>h_{\max }$ in the CKD model, whereas it is almost independent of $h_{0}$ (for $w=1$ ) or an increasing function of $h_{0}$ (for $w \geq 3$ ) in the DT model. This is probably the reason why the 1d DT model exhibits multiscaling over a longer period of time than the CKD model (in the 1d DT model, multiscaling lasts for at least six decades in time [8 10], compared to about three decades in the CKD model). This observation suggests that a controlled instability model in which the growth probability remains non-zero for large values of $h_{0}$ may exhibit multiscaling behavior over a longer period of time. It is difficult to construct such a model along the lines described in section IVA. This is because an increase in the value of $h_{\max }$, which can be achieved either by increasing $\lambda$ or by decreasing $c$, makes the instability strong, leading to deviations from scaling for global quantities such as $W$ (see, for example, Fig.15). We, therefore, constructed a different class of atomistic models in which the instability in the original KD model is controlled by an appropriate modification of the deposition rules. We describe below one such model and the results obtained from simulations of this model in one dimension.

The deposition rule in this model is designed to restrict the development of large values of the nearest-neighbor height difference. As in the KD model, the height variables in this model are discrete and time is measured in units of number of layers deposited. The deposition of a particle involves the following steps. A site is chosen at random and the KD rules (described in section II) are used to determine whether a particle is to be added to the chosen site or to one of its nearest neighbors. Let $n$ be the index of the site which is selected for the addition of a particle according to the KD rules and let $s_{l} \equiv\left|h_{n}-h_{n-1}\right|$ and $s_{r} \equiv\left|h_{n+1}-h_{n}\right|$ be the nearest-neighbor height differences at this site before the addition of the particle. The addition of a particle at site $n$ would change the values of both $s_{l}$ and $s_{r}$. If both $s_{l}$ and $s_{r}$ would decrease due to the addition of the particle at site $n$, the particle is added at site $n$ with probability one. If one of these two height differences would increase while the other one would decrease by the addition of a particle at site $n$, then we define $S$ to be the value of the height difference which would increase. If both the height differences increase due to the addition of a particle at site $n$, then we define $S$ to be the larger of $s_{l}$ and $s_{r}$. In these cases, the particle is deposited at site $n$ with probability $p=\exp (-u S)$, where $u$ is an adjustable parameter. Operationally, this is done by generating a random number $r$ which is distributed uniformly between 0 and 1 and the particle is deposited at site $n$ (i.e. the height variable at site $n$ is incremented by one) if $r \leq p$. If $r>p$, one of the nearest-neighbors of the site $n$ are chosen randomly and the particle is deposited there. This model reduces to the original KD model for $u=0$. For small positive values of $u$, the modification in the deposition rule disfavors but does not completely eliminate the growth of large nearest-neighbor height differences. There is a second route by which large height differences may form in this model. When a nearest-neighbor of the site $n$ is chosen for the deposition of a particle by the stochastic rule described above, it is not checked whether this deposition would increase the height differences between this site and its nearest neighbors. So, in some cases, the deposition of a particle at one of the nearest-neighbors of the site $n$ leads to an increase in the value of some nearest-neighbor height difference, and such increases are not controlled by the parameter $u$. One can, in principle, include such checks in the deposition rule, but 
this would make the rule very complicated and difficult to simulate efficiently.

Fig.23 shows our simulation results for the probability of growth of an isolated pillar of initial height $h_{0}$ in this model with $\lambda=2.0$ and $u=0.06$. The data shown were obtained by averaging over 5000 runs for systems with $L=$ 100. As expected, the probability of growth of isolated pillars in this model decreases with increasing $h_{0}$, but does not go to zero for large values of $h_{0}$. The magnitude of the growth probability for large values of $h_{0}$ in this model is similar to that in the 1d DT model, but the probability decays faster in time in the DT model. The observation of a non-zero probability of growth of pillars with large values of $h_{0}$ suggests that the time interval over which this model exhibits multiscaling behavior should be longer than that for the CKD model with similar parameter values. This expectation is confirmed by our simulation results which are shown in Figs. 24 and 25 for a system with $L=1000, \lambda$ $=2.0$ and $u=0.06$. The data shown represent averages over 200 runs. As can be seen in Fig.24, the time-dependence of the rms interface width $W$ shows excellent power-law scaling with $\beta \simeq 0.35$. The quantities $\left\{\sigma_{q}\right\}$, on the other hand, show evidence of multiexponent scaling over the entire time interval of these simulations. This time interval $\left(10^{4}\right.$ units) is an order of magnitude longer than the time interval over which multiscaling was observed in the CKD model (see Fig.16). The fact that the $\left\{\sigma_{q}\right\}$ with different values of $q$ do not grow in time with the same exponent is clearly shown in the inset of Fig.24 where we have plotted the time-dependence of the ratios $\sigma_{q} / \sigma_{1}$ for $q=2,3$ and 4 . The observed increase of the values of these ratios with time implies that $\left\{\sigma_{q}\right\}$ grows faster in time for larger values of $q$. It is perhaps more appropriate to characterize the observed behavior of the $\left\{\sigma_{q}\right\}$ as deviation from single-exponent scaling, rather than multiscaling. This is because $\log -\log$ plots of $\sigma_{q}$ vs $\tau$ (Fig.24) show substantial deviations from linear behavior. For this reason, it would not be particularly meaningful to define the exponents $\alpha_{q} / z$ in this model. As noted above, similar (but less pronounced) deviations from pure power-law behavior of the $\sigma_{q}$ s have also been observed in simulations of the 1d DT model. The data for the correlation functions $\left\{G_{q}(l)\right\}$ measured at time $\tau=$ $10^{4}$ are shown in Fig.25. These data show clear evidence of multiscaling, with exponent values $\zeta_{1} \simeq 0.79, \zeta_{2} \simeq 0.71$, $\zeta_{3} \simeq 0.62$, and $\zeta_{4} \simeq 0.57$. These results show that multiscaling similar to that observed in the 1d DT model can be generated by controlling the instability in the $1 \mathrm{~d} \mathrm{KD}$ model by appropriate modifications of the deposition rules.

A typical growth profile obtained in the modified KD model with $L=1000, \lambda=2.0$ and $u=0.06$ after the deposition of $10^{4}$ layers is shown in Fig.26. The similarity between this profile and the one shown in Fig.20 for the 1d DT model (after making the $h \rightarrow-h$ transformation) is quite remarkable. All the characteristic features present in the DT model profile are also present in the profile obtained in the modified KD model. This similarity provides additional support to the connection we propose between the DT model and the models with controlled instability studied in this paper. One could perhaps attempt to obtain better quantitative agreement between the multiscaling behavior in the DT and the controlled instability models by trying other variants of the LD equation and the KD model (e.g. by trying other forms for the function $f(x)$ in Eq. 19) for the CLD/CKD model or by modifying the deposition rules of the KD model in a different way). However, we do not see much purpose in such an attempt because (i) the multiscaling behavior is non-universal as we have been emphasizing throughout this paper, and (ii) such efforts are bound to be computationally intensive, the number of possible modifications of the LD equation and/or the KD model being arbitrarily large. We believe that the results presented in this paper unambiguously establish the (qualitative) connection we propose between controlled instability and the observed multiscaling in the 1d DT model.

\section{DISCUSSIONS}

In this section, we discuss a number of questions that are raised by the results obtained in our study. The work described here leads to two main results - the first one is about the presence of an instability in discretized growth equations and the second one is about the connection between this instability and multiscaling. Both these results have important implications in the study of models of surface growth. The observation of an instability in discretized versions of nonlinear growth equations raises the question of whether a similar instability is also present in the truly continuum limit. As discussed in section III above, the presence of an instability in the discretized version of the $1 \mathrm{~d}$ noiseless KPZ equation does not reflect the behavior of the corresponding continuum KPZ equation which does not have any instability. It is not clear whether the same conclusion would apply to the other growth equations (the KPZ equation with noise and the LD equation) considered here, mainly due to the fact that no exact result is available about the behavior of these continuum equations. The work of Ref. [20] suggests that the noiseless continuum LD equation in one dimension does not exhibit a true finite-time singularity in the sense that the height variable remains bounded by a value proportional to a power of the system size. That work, however, does not rule out the occurrence of an instability in which the height variable increases very rapidly in time at one or more isolated points in the system. In fact, the numerical results reported in Ref. [20] indicate that pillar-like structures initially grow in height in the continuum system also. Throughout this paper, we use the term "instability" to mean a rapid growth of the 
magnitude of the height variable in a local region of the system. The rate of change of the height in the region of the instability must be much faster than the corresponding rate in the background. The rapid change of the height in the instability region need not lead to a true divergence. The question of whether a true divergence occurs or not is interesting, but not very important for most practical purposes. For example, a rapid growth of the height variable at one or more points in the sample may lead to deviations from scaling behavior even if there is no true divergence. This is shown clearly in Fig.15 above. Also, it is almost impossible to distinguish in numerical work between a true divergence and a growth to a very large but finite value. The issue of a true divergence in the continuum growth equations is beyond the scope of our work.

Several atomistic models which are believed to belong to the universality class of the KPZ equation or the LD equation are known not to have any instability. For example, the restricted solid-on-solid model of Kim and Kosterlitz [22], which is believed to be in the same universality class as the KPZ equation, can not exhibit any instability of the kind being considered here because the nearest-neighbor height difference can not, by construction, exceed unity in this model. Similarly, the recently introduced conserved version [23] of the Kim-Kosterlitz model, which is supposed to belong to the universality class of the LD equation, also does not exhibit any instability. Another example is the model solved exactly by Gwa and Spohn [24 in one dimension. This model, believed to belong to the 1d KPZ universality class, is also known not to have any instability. These results, however, do not provide much help regarding the possibility of the occurrence of an instability in the corresponding continuum growth equations. This is because these models certainly differ from the corresponding continuum growth equations by virtue of the presence of terms which are irrelevant in the renormalization group sense. As shown in section IV above (and discussed in more detail below), the presence of such irrelevant terms may control or eliminate altogether the instability found in discretized growth equations. Therefore, the absence of an instability in models which are in the same universality class as the continuum growth equations does not imply that the growth equations do not have any instability.

It is clear from the discussion above that the possibility of occurrence of an instability in the continuum growth equations remains an open problem. We hope that our work will stimulate further investigation of this question. This question acquires special importance in view of the fact that a large part of the currently available information about the behavior of nonlinear growth equations has been obtained from studies of discretized versions. Our work, especially the results obtained here (and also in Ref. [21]) about the difference between the behavior of the discrete and continuum versions of the noiseless $1 \mathrm{~d}$ KPZ equation, raise serious questions about the applicability of the information obtained from numerical integration of discretized growth equations to the continuum equations. Our simulations suggest that the results obtained from numerical integration of the discretized equations should apply to the continuum case as long as there is no instability. This conclusion is suggested by the observation that the values of the "global" exponents $\beta, z$ and $\alpha$ extracted from the results of direct integration of discretized growth equations before the occurrence of the instability (or from runs in which no instability occurs, either due to the smallness of $\lambda$ or due to the imposition of control) are in good agreement (within error bars) with the exact (for the 1d KPZ equation) or expected (from e.g. renormalization group calculations for the LD equation) results for the continuum equation. We quote here the values of the exponent $\beta$ which is determined most accurately in our work. The calculated value of $\beta$ for the discretized LD equation in one dimension is $0.325 \pm 0.01$, and that for the discretized $1 \mathrm{~d} \mathrm{KPZ}$ equation is $0.32 \pm 0.02$. These values are in good agreement with the expected result, $\beta=1 / 3$, for the $1 \mathrm{~d}$ LD equation and the exact result, $\beta=1 / 3$, for the $1 \mathrm{~d} \mathrm{KPZ}$ equation. It would be useful to substantiate this conclusion with further study.

Our work also brings out the importance of terms which are often not included in continuum growth equations because they are irrelevant in the renormalization group sense. These terms involve higher powers of the gradient of the height variable. It is well-known that these terms are irrelevant in the KPZ equation in one dimension and in the LD equation in two and higher dimensions. This, however, does not mean that they are totally unimportant. The results described in section IV about the possibility of controlling the instability in the discretized growth equations by the introduction of higher powers of the gradient with appropriate coefficients indicate that such terms may play a very important role in the stability of the growth equations. These terms do not affect the values of the global exponents, but the growth equation may be unstable if such terms are not included. A recent paper by Marsili and Bray [25] makes a similar point. They consider an infinite-range version of the KPZ equation and show that this equation is unstable if certain higher-order terms (which are irrelevant in the RG sense) are not included. The instability they find is similar to the one we have found in discretized growth equations. There are well-known examples in equilibrium critical phenomena where formally irrelevant terms (the so-called "dangerous irrelevant variables") play a similar role in the control of instabilities. Consider, for example, the standard Ginzburg-Landau free energy functional [26] for a scalar order parameter (the so-called $\phi^{4}$ field theory). It is well-known 26] that the $\phi^{4}$ term in this free energy functional is irrelevant in the renormalization group sense if the dimension of space is higher than 4 . However, the sign of the coefficient $u$ of the $\phi^{4}$ term is very important because a negative value of $u$ leads to an instability. To control this instability, one needs to introduce one or more higher-order terms (typically, a $\phi^{6}$ term) which are also irrelevant in the renormalization group sense. The role played by the $\phi^{6}$ term in this example is qualitatively similar to the role of the higher-order terms introduced in our models of controlled instability. Another aspect of this example 
[26] from equilibrium critical phenomena may also be relevant to the growth problems being considered here. The Ginzburg-Landau model is known to exhibit strong crossover effects if it is close to an instability i.e. if the value of $u$ is positive but very small, the free energy density being singular in the limit $u \rightarrow 0$. This crossover in this model is from apparent tricritical behavior (which would be the asymptotic critical behavior for $u=0$ ) to the usual critical behavior expected for $u>0$. These crossover effects lead to apparent non-universal values of the effective critical exponents, and the correct values of the critical exponents are observed only very close to the critical point (i.e. at very long length scales). As discussed below, there are reasons to believe that the non-universal behavior observed in some of the growth models studied in our work is also a consequence of crossover effects arising from the proximity of the system to an instability. It will be particularly interesting to try to establish formally this suggestive but qualitative analogy between anomalous dynamic scaling in DT and controlled instability models and crossover scaling induced by dangerous irrelevant variables in equilibrium critical phenomena.

The observation that the instability in discretized growth equations can be effectively controlled or eliminated altogether by the introduction of formally irrelevant higher-order terms suggests a practical solution to the numerical problems encountered in previous studies [12,13] using direct integration. If the discretized version of a continuum growth equation belongs in the same universality class as the continuum equation itself, then a direct numerical integration of a version of the discrete equation which is controlled by the introduction of irrelevant higher order terms should yield values of global critical exponents appropriate for the continuum growth equation without running into instability problems. The usefulness of this method is illustrated by the results described in section IVA for models in which the instability is controlled or eliminated. It is interesting to note that the prescription suggested by Newman and Bray [21] for getting rid of the instability in the discretized version of the noiseless 1d KPZ equation, while differing in details, also amounts to the introduction of terms involving higher powers of the gradient of the height variable.

The $1 \mathrm{~d}$ LD equation is special in the sense that the terms involving higher powers of the gradient are all marginally relevant in one dimension. It has been argued in Ref. [10] that the marginal relevance of these terms may lead to nonuniversal corrections to the critical exponents and also to anomalous dynamic scaling where local and global exponents differ [14]. The value of the exponent $\beta$ found in our simulations of the CKD model and the modified KD model described in section IVB is $0.355 \pm 0.005$, which is close to, but significantly different from the value $1 / 3$ expected from renormalization group calculations [19] on the LD equation. It is tempting to attribute this difference to the presence of higher powers of the gradient in the controlled KD models. However, we do not have any other evidence to back up this explanation which, therefore, remains speculative. The leading order mode-coupling analysis of Ref. [10] also suggests that the marginal relevance of the higher order terms may play an important role in the multiscaling behavior. As described in section IVA, we do find approximate multiscaling in models in which the instability is controlled by the introduction of an infinite series of higher powers of the gradient with appropriate coefficients. Therefore, there is a definite connection between multiscaling and the coefficients of the higher order terms. However, the values of the effective multiscaling exponents $\alpha_{q}$ and $\zeta_{q}$ obtained from our simulations do not satisfy the specific quantitative prediction of Ref. [10]. Thus, on a conceptual level, there is a correspondence between our finding of a ("controlled") instability and the infrared singularity underlying the work of Ref. [10]. However, a more detailed connection between these two works must await further investigation.

The possibility that continuum and discretized versions of a growth equation may exhibit different behavior brings up the question of which version is more appropriate for describing real physical systems. While we do not claim to have an answer to this question, we wish to point out that it may be inappropriate to regard the discretized version of a growth equation to be less "fundamental" than the continuum version. Discretized versions of nonlinear growth equations may actually be closer to the physics of growth processes than the continuum equations. This is because all growth processes are discrete at the atomic scale due to the presence of the cutoff introduced by the atomic lattice structure. A continuum description is obtained under certain assumptions about the smoothness of the growth profile. The discreteness at the atomic level is incorporated in the continuum description through the introduction of a short-distance cutoff. The instability we find suggests that the assumptions which go into the development of a continuum description may not be valid under certain circumstances, depending on the values of the bare coupling constants, cutoffs etc.

Finally, we discuss the implications of our results on the origin and nature of multiscaling in models of surface growth. It is clear from the results described in section IV that a controlled instability is responsible for the multiscaling behavior in the models we have studied. In the CKD model described in section IVA, multiscaling is found only if the value of the control parameter $c$ is such that the instability is present. Also, multiscaling behavior for such values of $c$ is observed only during the time interval over which the instability is operative. The same behavior is found for the modified KD model described in section IVB. The multiscaling behavior exhibited by these models is very similar to that observed [8 10] in the 1d DT model and in other related atomistic models of surface growth. As described in section IVB, the behavior of the probability of growth of an isolated groove as a function of time in the $1 \mathrm{~d}$ DT model is similar to that found in the modified KD model for appropriate values of the control parameter $u$. All these 
results clearly establish a connection between multiscaling in growth models and a controlled instability of the kind described here. Our study also leads to the important conclusion that the multiscaling found in growth models is necessarily non-universal and transient in time. Consider, for example, the CKD model described in section IVA. In this model, the values of the exponents $\beta, z$ and $\alpha$, which describe the global properties of the growing interface, are essentially the same for all values of the control parameter $c$ (of course, the value of $c$ should be sufficiently large so that the rms surface width $W$ does not show any departure from power-law behavior; otherwise, these exponents can not be defined). The models with different values of $c$, therefore, belong to the same universality class as far as the global behavior is concerned. On the other hand, the multiscaling behavior, which involves local quantities because it is manifested in the time-dependence of the moments of the nearest-neighbor height difference and in the dependence of the correlation functions $G_{q}(l, \tau)$ on $l$ for $l<\xi(\tau)$, is found to be very different for different values of $c$. Similar results are obtained for the modified KD model of section IVB. In this model also, the global exponents are found to be insensitive to the value of the control parameter $u$, but the multiscaling behavior depends crucially on it. These results clearly show that multiscaling in these models is a non-universal feature. We also find that all the models studied in our work exhibit multiscaling only over a limited period of time. The length of the time interval over which multiscaling is observed varies greatly from one model to another, but multiscaling is found to disappear at sufficiently long times in all these models. This is not a saturation effect because the rms surface width $W$ continues to grow beyond the time at which multiscaling disappears (see Fig.16, for example). A careful look at the simulation data of Ref. 88 10] shows that the feature of non-universality and the transient nature of multiscaling are present in varying degrees in all the atomistic models studied in these papers. It is, therefore, reasonable to conclude that multiscaling in discrete growth models is a non-universal transient behavior, possibly related to the presence of higherorder terms involving higher powers of the gradient of the height variable in the continuum equations appropriate for these discrete models. The similarity between our results for the controlled-instability versions of the KD model (which, by construction [7], provides an atomistic version of the LD growth equation) and those obtained in Ref. 8 10. for the 1d DT and related models suggests that the latter models are described by the LD equation with the addition of terms containing higher powers of the height gradient with appropriate coefficients. The marginality of these terms in one dimension may provide an explanation of why the time period over which multiscaling is observed in the $1 \mathrm{~d}$ DT model is very long. It is interesting to note in this context that recent simulations of the DT model in two dimensions [27] show that this system also exhibits transient multiscaling, but over a much shorter interval of time. This may be related to the fact that terms involving higher powers of the height gradient are marginally irrelevant in the $2 \mathrm{~d}$ LD equation. Further investigation of the role of these higher-order terms in the behavior of growth equations would be interesting and important for a complete understanding of the problem.

This work is supported by the US-ONR and the NSF-DMR-MRG. CD and JMK would like to thank the Condensed Matter Physics Group of the University of Maryland for hospitality. One of the authors (JMK) also wishes to thank Hallym Academy of Science, Hallym University for support.

* $\quad$ Present and permanent address: Dept. of Physics, Indian Institute of Science, Bangalore 560012, India.

** Present and permanent address: Dept. of Physics and Interdisciplinary Research Center in Physical Science, Hallym University, Chunchon, 200-702, Korea.

[1] A.-L. Barabasi and H. E. Stanley, Fractal Concepts in Surface Growth (Cambridge University Press, Cambridge, 1995).

[2] T. Hapin-Healy and Y.-C. Zhang, Phys. Rep. 254, 216 (1995); J. Krug, Adv. Phys. (to be published).

[3] M. Kardar, G. Parisi and Y.-C. Zhang, Phys. Rev. Lett. 56, 889 (1986).

[4] Z. W. Lai and S. Das Sarma, Phys. Rev. Lett. 66, 2348 (1991).

[5] J. Villain, J. Phys. I (France) 1, 19 (1991); D.E. Wolf and J. Villain, Europhys. Lett. 13, 389 (1990).

[6] S. Das Sarma and P. Tamborenea, Phys. Rev. Lett. 66, 325 (1991); P.I. Tamborenea and S. Das Sarma, Phys. Rev. E 48, 2599 (1993).

[7] J. M. Kim and S. Das Sarma, Phys. Rev. Lett. 72, 2903 (1994).

[8] J. Krug, Phys. Rev. Lett. 72, 2903 (1994).

[9] S. Das Sarma, C. J. Lanczycki, R. Kotlyar and S. V. Ghaisas, Phys. Rev. E 53, 359 (1996).

[10] J. K. Bhattacharjee, S. Das Sarma and R. Kotlyar, Phys. Rev E 53, R1313 (1996).

[11] D. Lohse and S. Grossmann, Physica A 194, 519 (1993).

[12] Y. Tu, Phys. Rev. A 46, R729 (1992).

[13] K. Moser, J. Kertesz and D.E. Wolf, Physica A 178, 215 (1991); J.P. Doherty et al., Phys. Rev. Lett. 72, 2041 (1994).

[14] S. Das Sarma, S. V. Ghaisas and J. M. Kim, Phys. Rev. E 49, 122 (1994). 
[15] J. P. Eckmann and I. Procaccia, Phys. Lett. A 182, 93 (1993).

16] C. Dasgupta, S. Das Sarma and J. M. Kim, "Controlled Instability and Multiscaling in Models of Epitaxial Growth", submitted to Phys. Rev. Lett. (MS Code \# LP5640, received on 2 February, 1996).

[17] J. M. Kim and S. Das Sarma, Phys. Rev. B 51, 1889 (1995).

[18] Handbook of Mathematical Functions, edited by M. Abramowitz and A. Stegun, Dover, New York, 1970, pp 883-884.

[19] S. Das Sarma and R. Kotlyar, Phys. Rev. E 50, R4275 (1994).

[20] V. Putkaradze, T. Bohr and J. Krug, preprint.

[21] T. J. Newman and A. J. Bray, preprint.

[22] J. M. Kim and J. M. Kosterlitz, Phys. Rev. Lett. 62, 2289 (1989); J. M. Kim, J. M. Kosterlitz and T. Ala-Nissila, J. Phys. A 24, 5569 (1991).

[23] Y. Kim, D. K. Park and J. M. Kim, J, Phys. A 27, L533 (1994); Y. Kim and J. M. Kim, J. Kor. Phys. Soc. 28, S424 (1995).

[24] L.-H. Gwa and H. Spohn, Phys. Rev. A 46, 844 (1992).

[25] M. Marsili and A. J. Bray, Phys. Rev. Lett. 73, 2750 (1996).

[26] S.-K. Ma, Modern Theory of Critical Phenomena (Benjamin, Reading, 1976). See, in partcular, p. 179-188 of chapter VII.

[27] P. Puniyndu, unpublished and private communications.

FIG. 1. The rms interface width $W$ and the moments $\sigma_{q}, q=1,4$, of the nearest-neighbor height difference (see text) as functions of time $\tau$ for the $1 \mathrm{~d}$ discretized LD equation with $\lambda=1.0$. The data shown were obtained for a system with $L=$ $10^{4}$, using an integration time step $\Delta \tau=0.01$. Inset: The height-difference correlation functions $G_{q}(l), q=1,4,($ see text) as functions of the separation $l$ for this system at time $\tau=10^{4}$.

FIG. 2. The rms interface width $W$ and the moments $\sigma_{q}, q=1,4$, of the nearest-neighbor height difference as functions of time $\tau$ for the $1 \mathrm{~d}$ KD model with $\lambda=0.5$. The data shown represent an average over 10 runs on systems with $L=10^{4}$. Inset: The height-difference correlation functions $G_{q}(l), q=1,4$, as functions of the separation $l$ for this system at time $\tau=10^{4}$.

FIG. 3. The rms interface width $W$ and the moments $\sigma_{q}, q=1,4$, of the nearest-neighbor height difference as functions of time $\tau$ for the $1 \mathrm{~d}$ KD model with $\lambda=1.0$. The data shown represent an average over 10 runs on systems with $L=10^{4}$.

FIG. 4. Growth probability (see text) of a pillar in the $1 \mathrm{~d}$ discretized LD equation $(\lambda=1.0, \Delta \tau=0.01,2000$ runs on $L=$ 100 samples) as a function of time $\tau$. Results for three different values, 14, 17 and 20, of the initial height $h_{0}$ are shown.

FIG. 5. Growth probability of a pillar of initial height $h_{0}$ in the 1 d discretized LD equation $(\lambda=1.0,2000$ runs on $L=$ 100 samples) at time $\tau=1$ as a function of $h_{0}$, calculated with three different values, $0.01,0.001$ and 0.0001 , of the integration time step $\Delta \tau$.

FIG. 6. The dependence of $h_{c}$, the critical height of a pillar (or the depth of a groove, see text), on the coupling constant $\lambda$ in the discretized LD equation in one and two dimensions and the discretized KPZ equation in one dimension. The results shown were obtained from numerical integrations with $\Delta \tau=0.01$. The solid lines are the best fits to the form $h_{c}(\lambda)=A / \lambda$.

FIG. 7. Development of the instability induced in the $1 \mathrm{~d}$ discretized LD equation $(\lambda=1.0)$ by the presence of a pillar of initial height $h_{0}=25$. Interface profiles at times $\tau=0.05,0.1,0.15$ and 0.17 , obtained for a $L=100$ system using $\Delta \tau=10^{-4}$, are shown. In the initial state, the height is zero everywhere except at the 50th site where the height is $h_{0}$.

FIG. 8. Development of the instability induced in the 1d discretized KPZ equation $(\lambda=1.0)$ by the presence of a groove of initial depth $h_{0}=30$. Interface profiles at times $\tau=0.1,0.3$ and 0.5 , obtained for a $L=100$ system using $\Delta \tau=10^{-4}$, are shown. In the initial state, the height is zero everywhere except at the 50 th site where the height is $-h_{0}$.

FIG. 9. Probability of instability (see text) induced by a groove of initial depth $h_{0}$ in the $1 \mathrm{~d}$ discretized KPZ equation ( $\lambda$ $=1.0,2000$ runs with $\Delta \tau=0.01$ on $L=100$ samples) as a function of time $\tau$. Results for three different values, 24, 25 and 26 , of the initial height $h_{0}$ are shown. 
FIG. 10. Time dependence of the maximum nearest-neighbor height difference $s_{\max }$ in the $1 \mathrm{~d}$ discretized LD equation $(\lambda$ $=4, \Delta \tau=0.01, L=1000,200$ runs $)$, the $2 \mathrm{~d}$ discretized LD equation $(\lambda=5, \Delta \tau=0.01$, system size $=200 \times 200,30$ runs $)$ and the $1 \mathrm{~d}$ discretized $\mathrm{KPZ}$ equation $\left(\lambda=5, \Delta \tau=0.01, L=10^{4}, 200\right.$ runs). The solid line is a fit of the data for the $1 \mathrm{~d}$ LD equation to the form $s_{\max }(\tau)=a+b \ln \tau$.

FIG. 11. The average instability time $\tau_{\text {ins }}$ (see text) as a function of $\lambda$ for the $1 \mathrm{~d}$ discretized LD equation $(L=1000, \Delta \tau=$ 0.01 , number of runs $=150,200,500,1000,1000$ for $\lambda=3,4,5,6,7$, respectively) and the $1 \mathrm{~d}$ discretized KPZ equation $(L=$ $10^{4}, \Delta \tau=0.01,200$ runs for each value of $\left.\lambda\right)$. The arrow indicates a lower bound for $\tau_{\text {ins }}$ for the KPZ equation with $\lambda=4$. The solid line is a fit of the LD equation data for $\lambda=4,5$ and 6 to the form $\tau_{\text {ins }}=A e^{B / \lambda^{2}}$.

FIG. 12. The ratios $\sigma_{q}(\tau) / \sigma_{1}(\tau), q=2,3,4$ and 5, as functions of time $\tau$ for the $1 \mathrm{~d}$ discretized LD equation with $\lambda=4.0$, $L=1000, \Delta \tau=0.01$, averaged over 100 runs. The observed growth of the values of these ratios with time indicates deviations from single-exponent scaling.

FIG. 13. The dependence of the quantity $\Delta$, which measures the initial rate of growth of the height of an isolated pillar in the $1 \mathrm{~d}$ CLD model without noise, on $h_{0}$, the initial height of the pillar. The results shown are for $\lambda=4.0$ and three different values, 0.02, 0.03, and 0.3, of the control parameter $c$ (see text).

FIG. 14. The probability of growth of an isolated pillar in the $1 \mathrm{~d}$ CKD model $(\lambda=2.0, c=0.02)$ as a function of time. These data were obtained from 2000 runs on samples with $L=100$. Results for three different values, 5.0, 25.0 and 45.0, of the initial height $h_{0}$ are shown.

FIG. 15. The rms interface width $W$ as a function of time $\tau$ for the $1 \mathrm{~d}$ CLD model $(\lambda=4.0, L=1000, \Delta \tau=0.01)$ with two values of $c(10$ runs for $c=0.02$ and 40 runs for $c=0.05)$, and for the $1 \mathrm{~d}$ CKD model $(\lambda=2.0, L=1000)$ with two values of $c$ (200 runs for $c=0.02$ and 10 runs for $c=0.005)$.

FIG. 16. The rms interface width $W$ and the moments $\sigma_{q}, q=1-4$, of the nearest-neighbor height difference as functions of time $\tau$ for the $1 \mathrm{~d}$ CKD model $\left(\lambda=2, c=0.02, L=1000\right.$, averaged over 2000 runs). Inset: The ratios $\sigma_{q}(\tau) / \sigma_{1}(\tau), q=2$, 3 and 4 , as functions of time $\tau$. The data shown in the inset were averaged over 200 runs.

FIG. 17. The height-difference correlation functions $G_{q}(l), q=1-4$, at time $\tau=1000$ as functions of the separation $l$ for the 1d CKD model $(\lambda=2, c=0.02, L=1000$, averaged over 2000 runs). The solid lines are power-law fits to the data for $l \leq$ 10. Inset: The ratios $G_{q}(l) / G_{1}(l), q=2,3$ and 4 as functions of the separation $l$.

FIG. 18. Scaling plot for the dependence of the interface width $W$ in the $1 \mathrm{~d}$ CKD model $(\lambda=2.0, c=0.02)$ on time $\tau$ for systems with different sizes $L$. The data for $L=20,40$ and 80 were obtained by averaging over 2000,1000 and 1000 runs, respectively. The values of the exponents used in this scaling plot are: $\alpha=1.35, \beta=0.355$.

FIG. 19. Distribution of the nearest-neighbor height difference $s$ in the $1 \mathrm{~d}$ CKD model $(\lambda=2.0, c=0.02)$. Results are shown for $L=1000, \tau=1000$ (averaged over 2000 runs) and also for $L=80$ in the saturation regime $\left(5 \times 10^{4} \leq \tau \leq 5 \times 10^{5}\right)$, averaged over 1000 runs. The solid lines represent fits of the data to the power-law form, $P(s) \propto s^{-\eta}$, with $\eta=3.2$ for the $L$ $=1000$ data and $\eta=2.5$ for the $L=80$ data. The inset shows the best stretched exponential fit to the $L=80$ data for small values of $s$.

FIG. 20. Typical profile of the interface generated at time $\tau=10^{4}$ in the $1 \mathrm{~d}$ DT model with $L=1000$. The quantity plotted along the vertical axis is the deviation of the height from the average height which, by definition, is equal to $\tau$. 
FIG. 21. Typical profile of the interface generated at time $\tau=10^{4}$ in the $1 \mathrm{~d}$ CKD model with $\lambda=2.0, c=0.02$ and $L=$ 1000. The average height which, by definition, is equal to $\tau$, has been subtracted from the values plotted along the vertical axis.

FIG. 22. The probability of increase of the depth of an isolated groove of width $w=1$ in the $1 \mathrm{~d}$ DT model as a function of time $\tau$. The data were obtained by averaging over $10^{5}$ runs on samples with $L=32$. This probability is independent of the initial depth $h_{0}$ of the groove for all $h_{0}>10$. Inset: The probability of increase of the depth of an isolated groove of width $w=$ 3 in the 1d DT model. Results obtained by averaging over 1000 runs on $L=128$ samples are shown for initial depth $h_{0}=30$ and $h_{0}=90$.

FIG. 23. The probability of growth of an isolated pillar in the $1 \mathrm{~d}$ modified KD model (see text) with $\lambda=2.0, u=0.06$, as a function of time. These data were obtained from 2000 runs on samples with $L=100$. Results for four different values, 10 , 30,50 and 70 , of the initial height $h_{0}$ are shown.

FIG. 24. The rms interface width $W$ and the moments $\sigma_{q}, q=1-4$, of the nearest-neighbor height difference as functions of time $\tau$ for the $1 \mathrm{~d}$ modified $\mathrm{KD}$ model $(\lambda=2, u=0.06, L=1000$, averaged over $200 \mathrm{runs})$. Inset: The ratios $\sigma_{q}(\tau) / \sigma_{1}(\tau)$, $q=2,3$ and 4 , as functions of time $\tau$.

FIG. 25. The height-difference correlation functions $G_{q}(l), q=1-4$, at time $\tau=10^{4}$ as functions of the separation $l$ for the $1 \mathrm{~d}$ modified KD model $(\lambda=2, u=0.06, L=1000$, averaged over 200 runs).

FIG. 26. Typical profile of the interface generated at time $\tau=10^{4}$ in the $1 \mathrm{~d}$ modified KD model with $\lambda=2.0, u=0.06$ and $L=1000$. The quantity plotted along the $y$-axis is the deviation of the height from the average height which, by definition, is equal to $\tau$. 


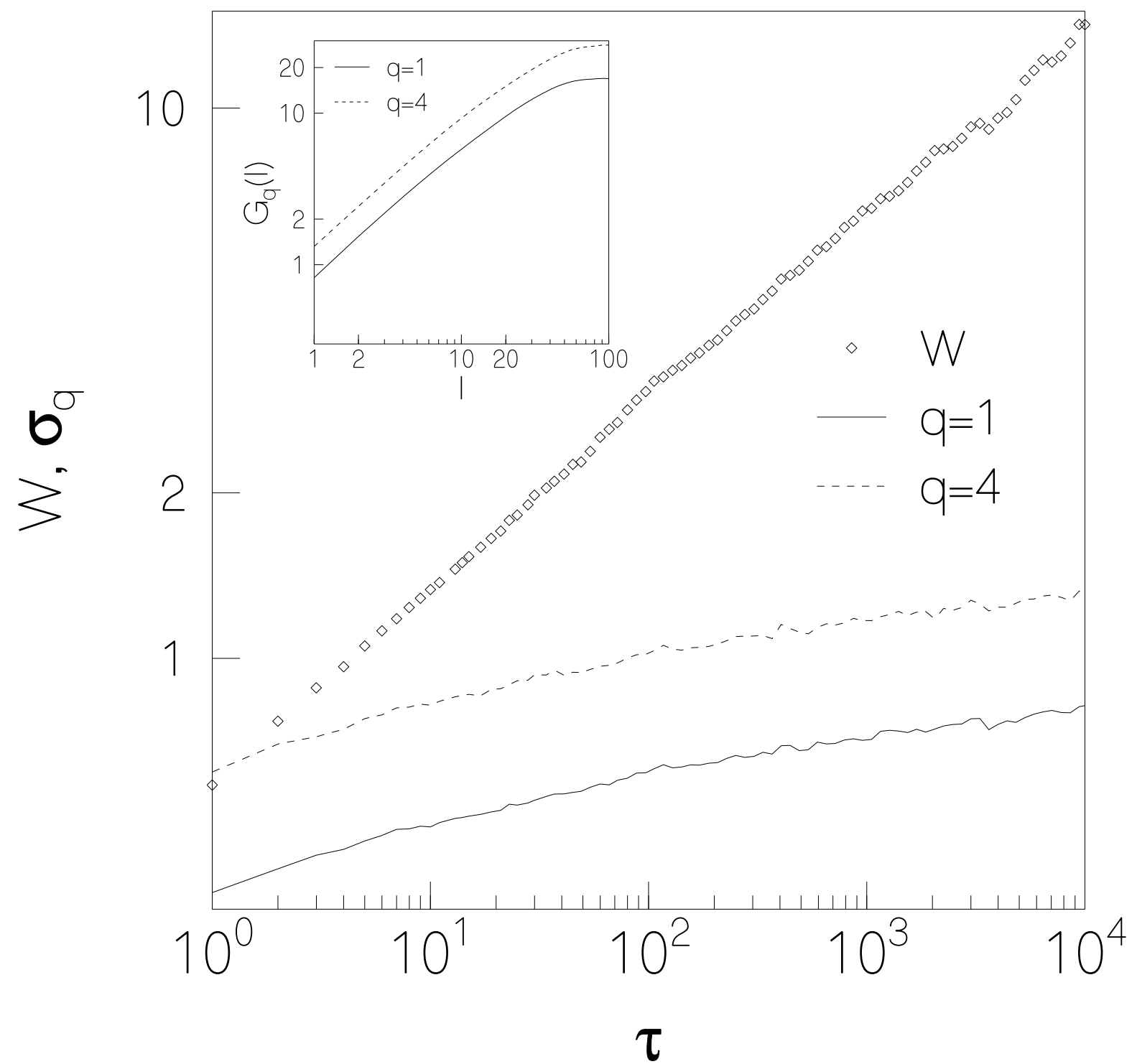




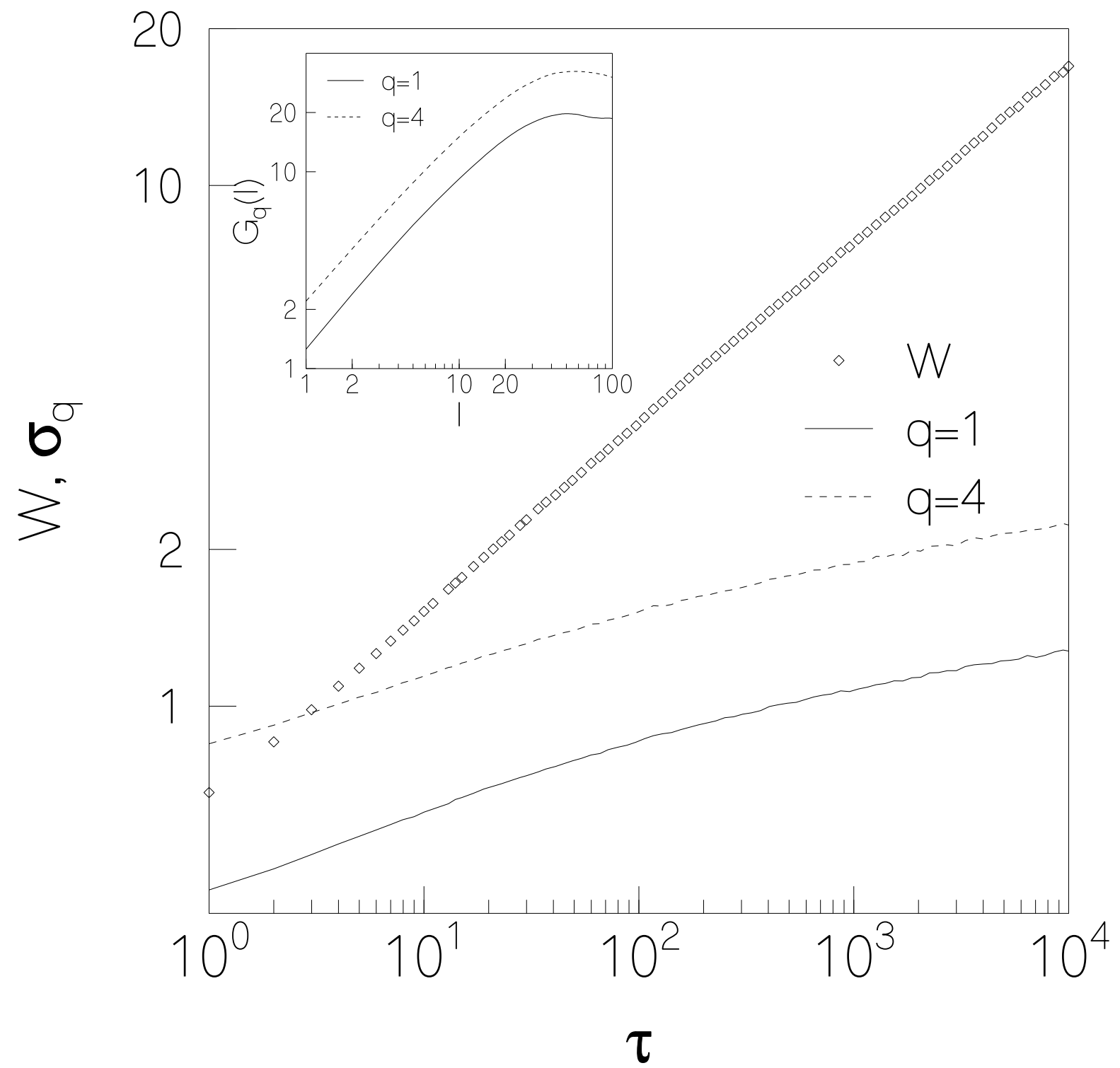




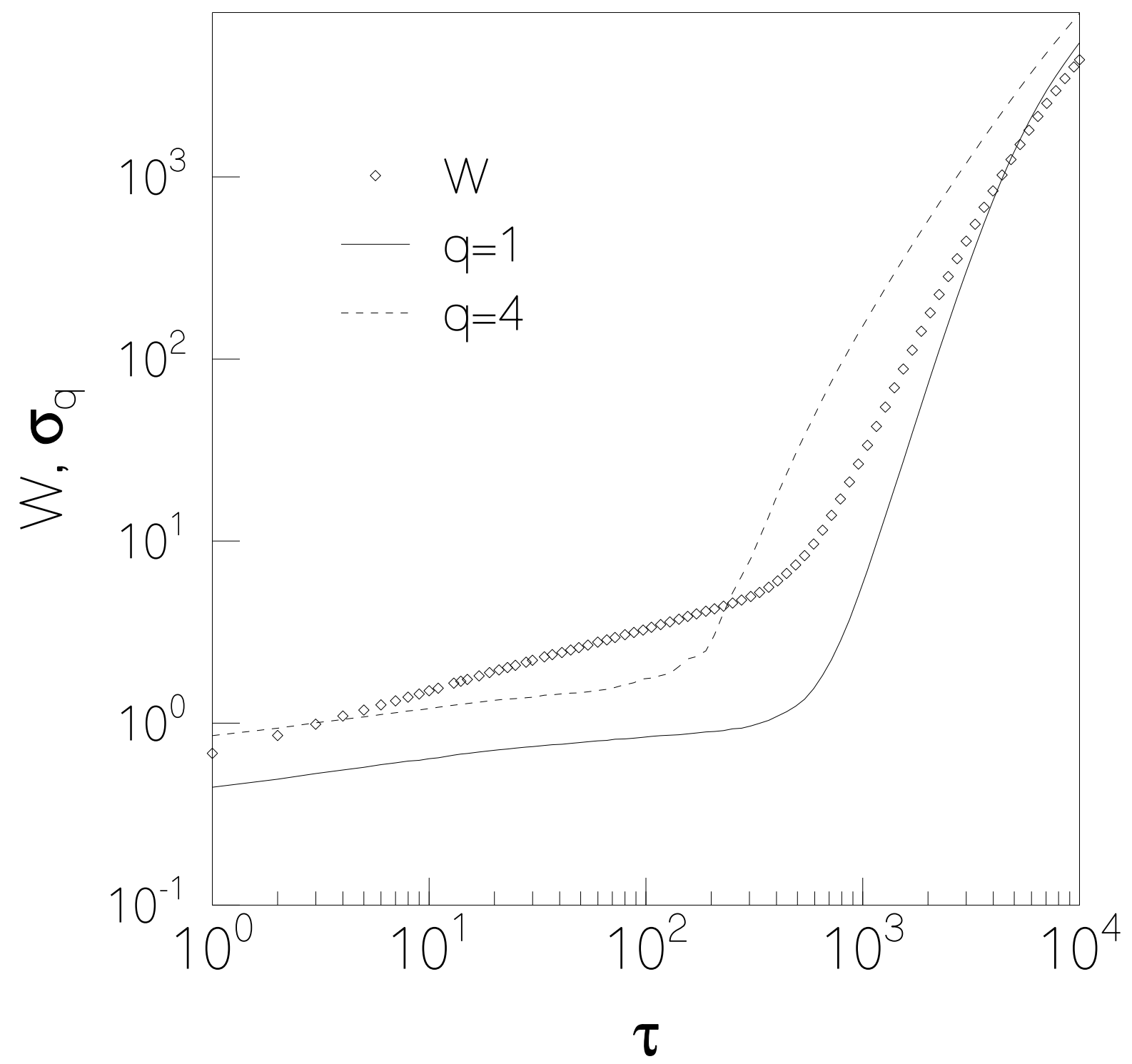




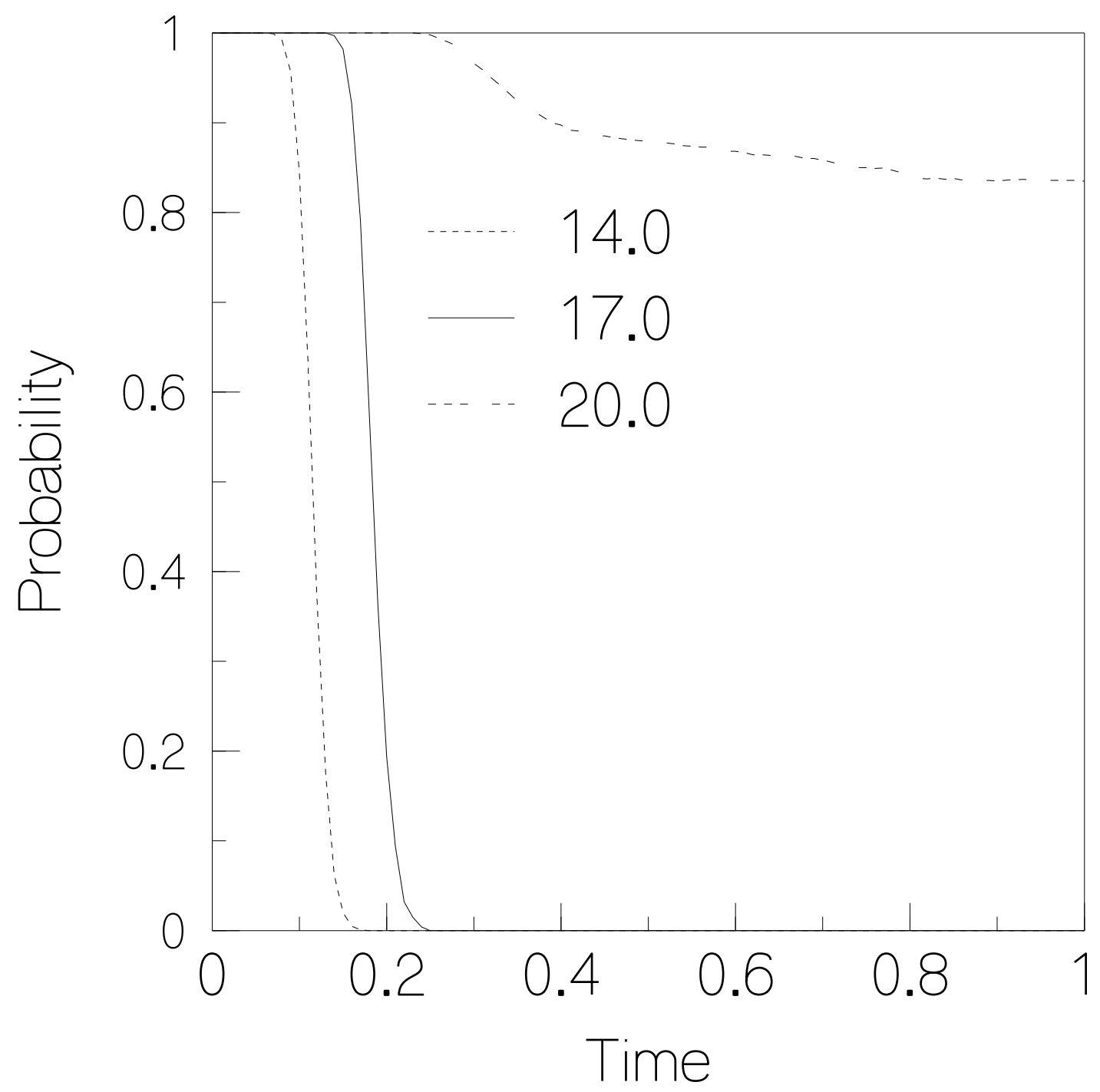




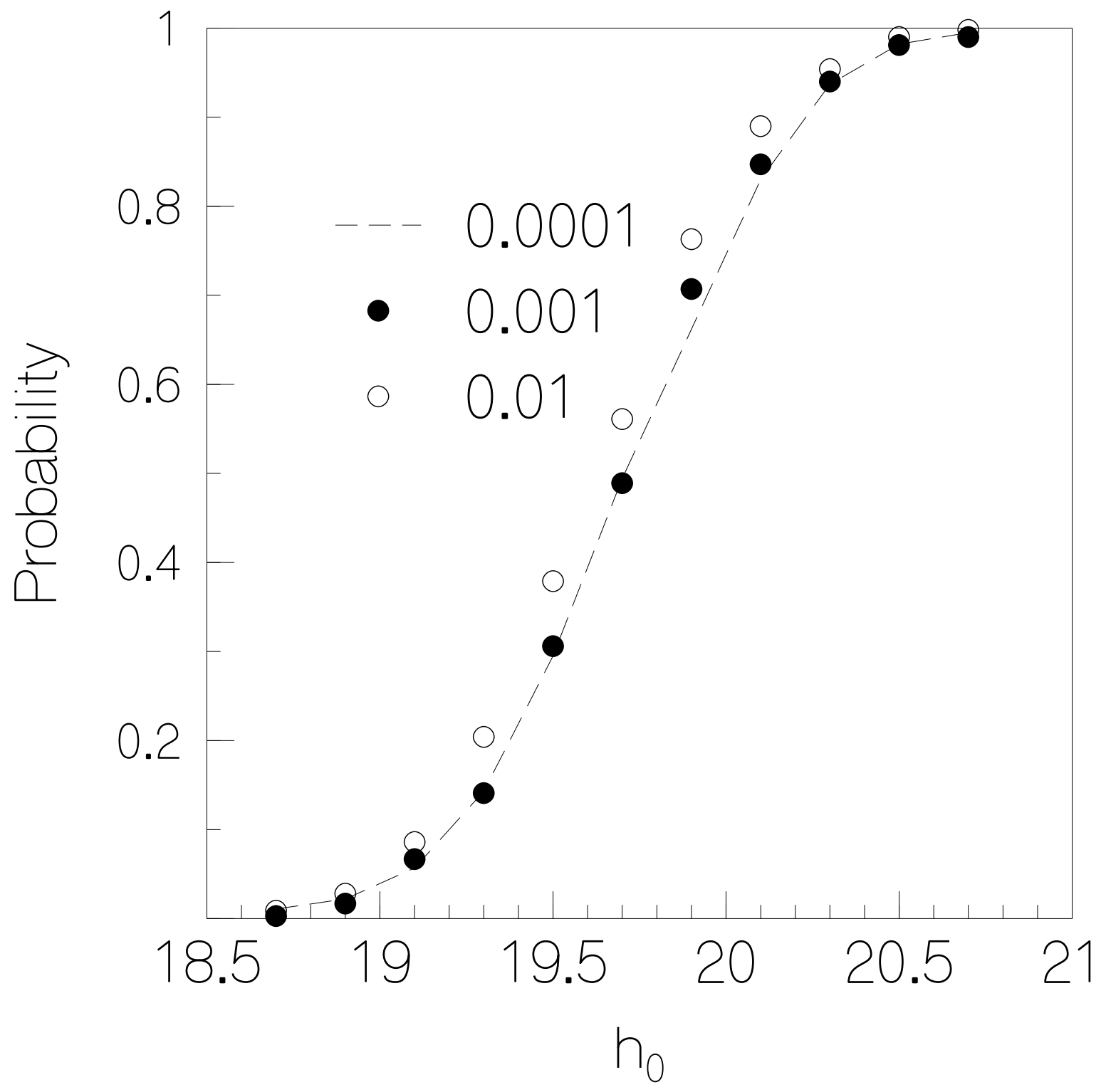




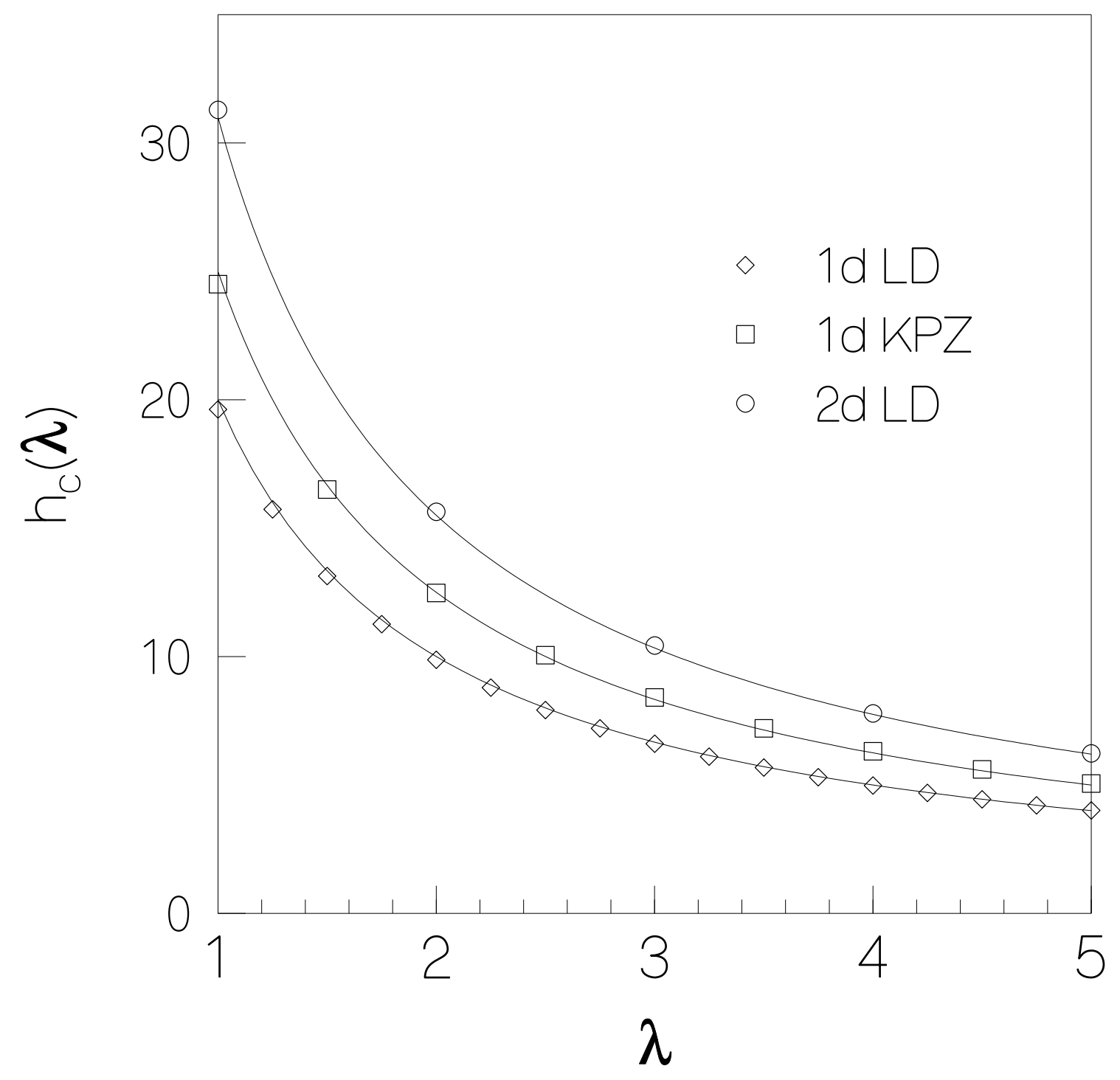




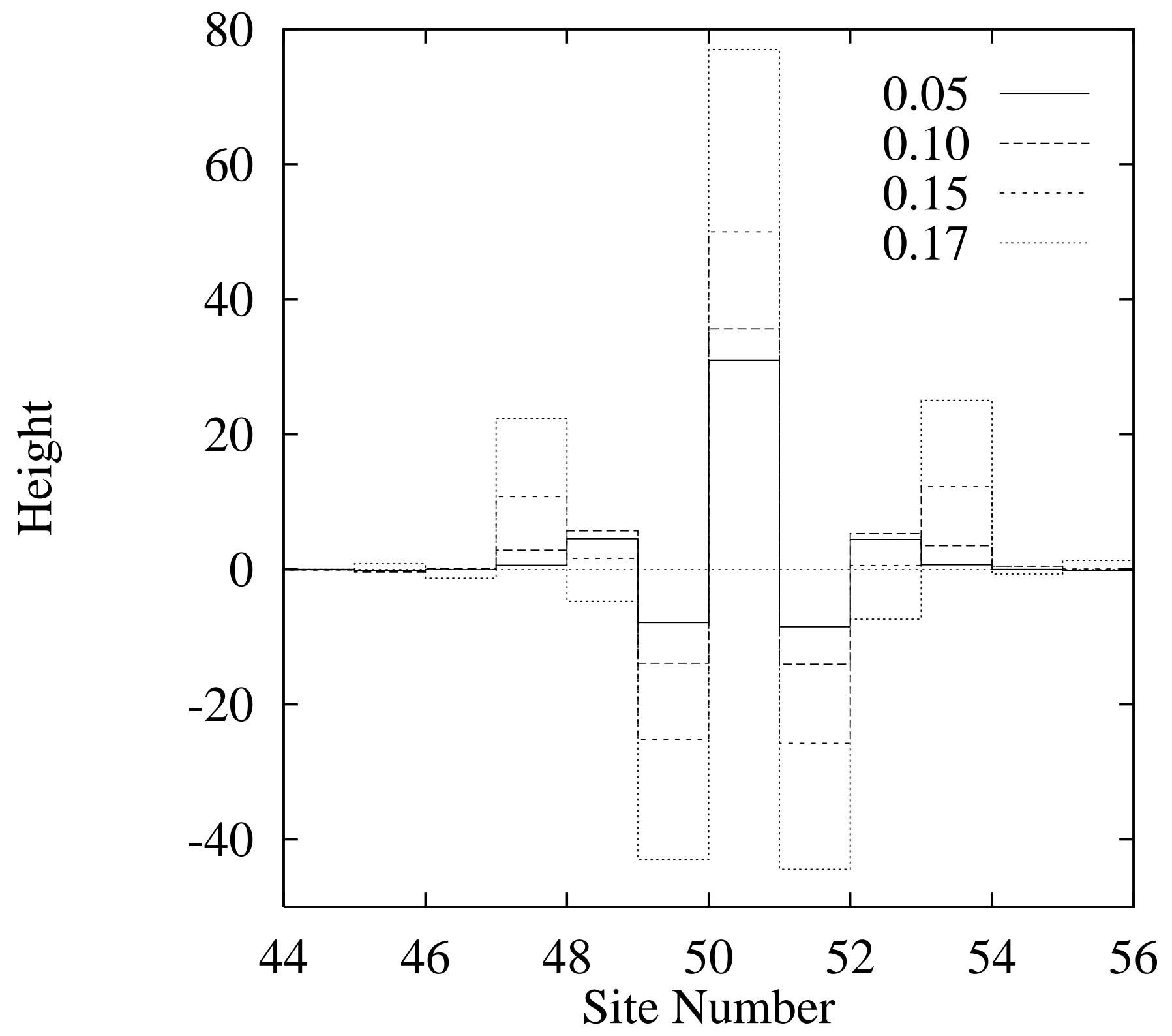




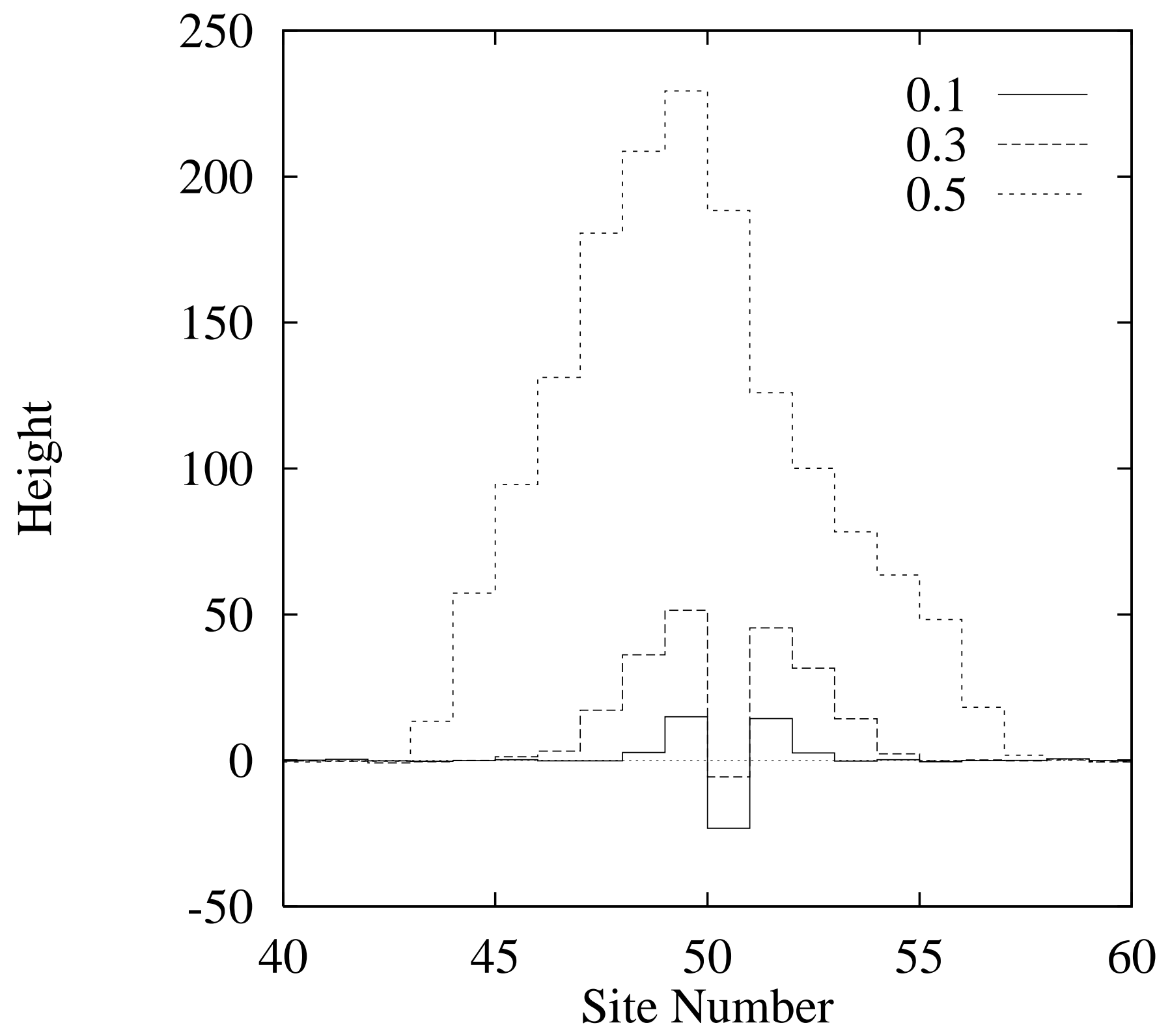




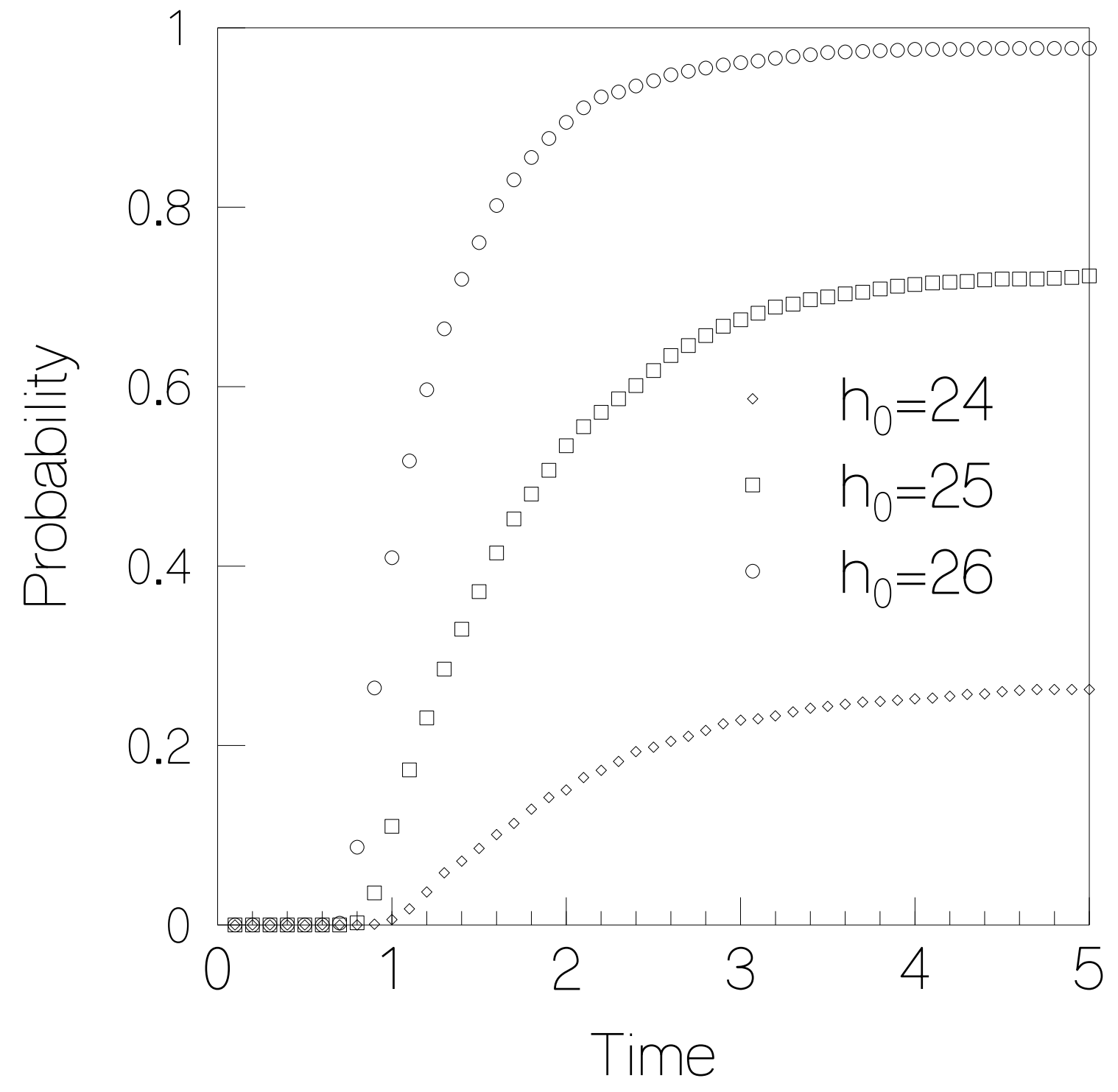




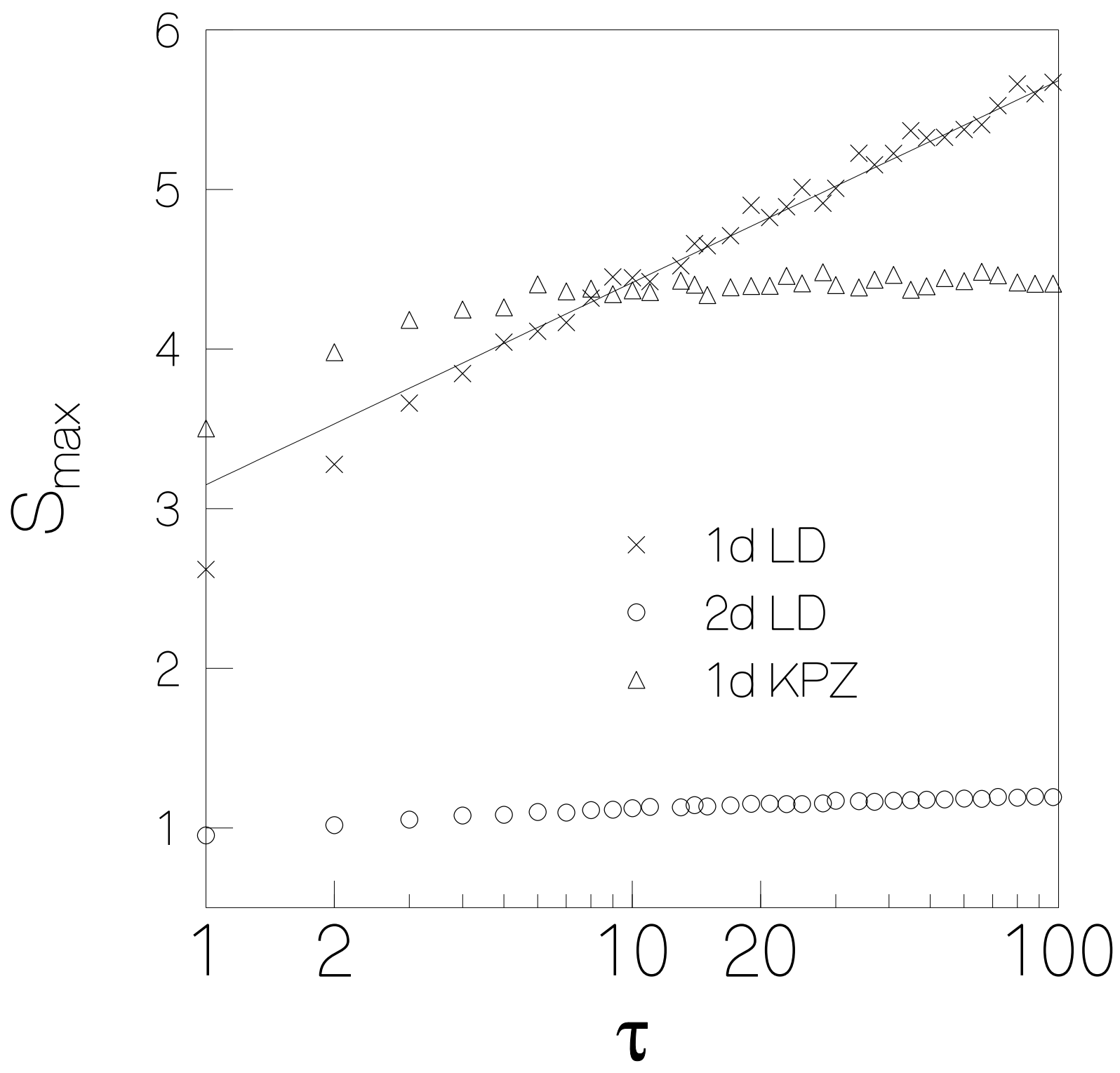




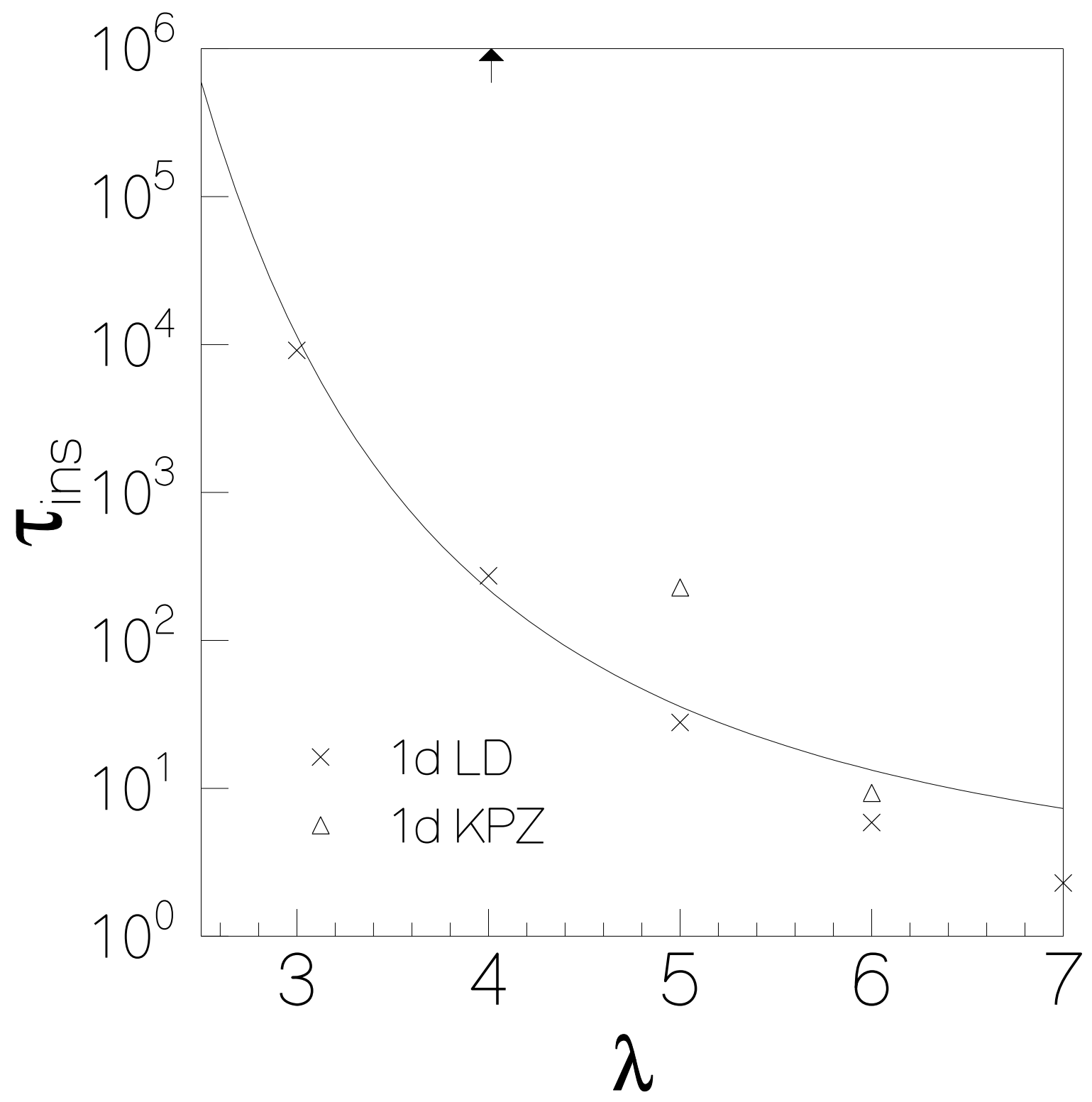




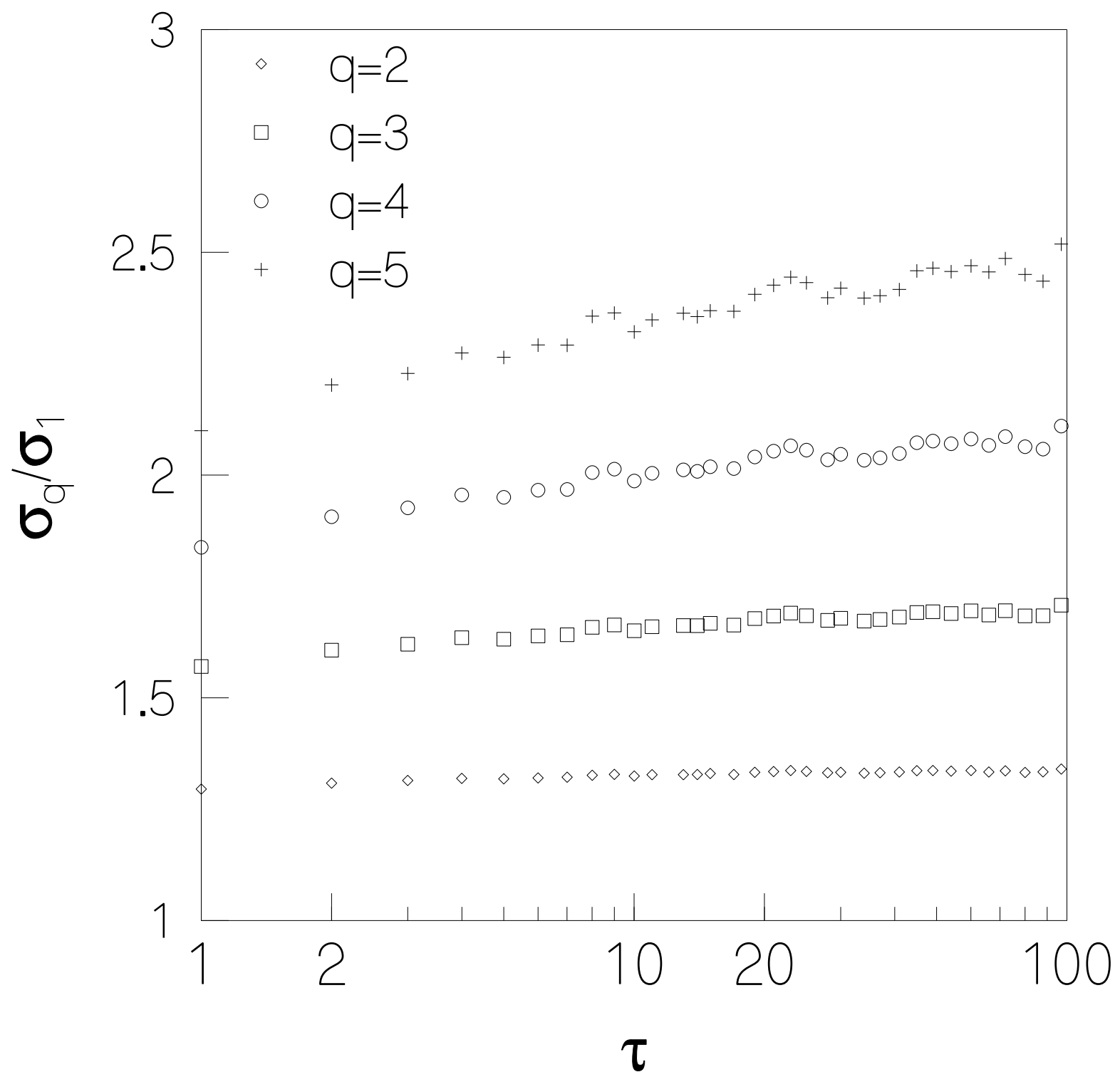




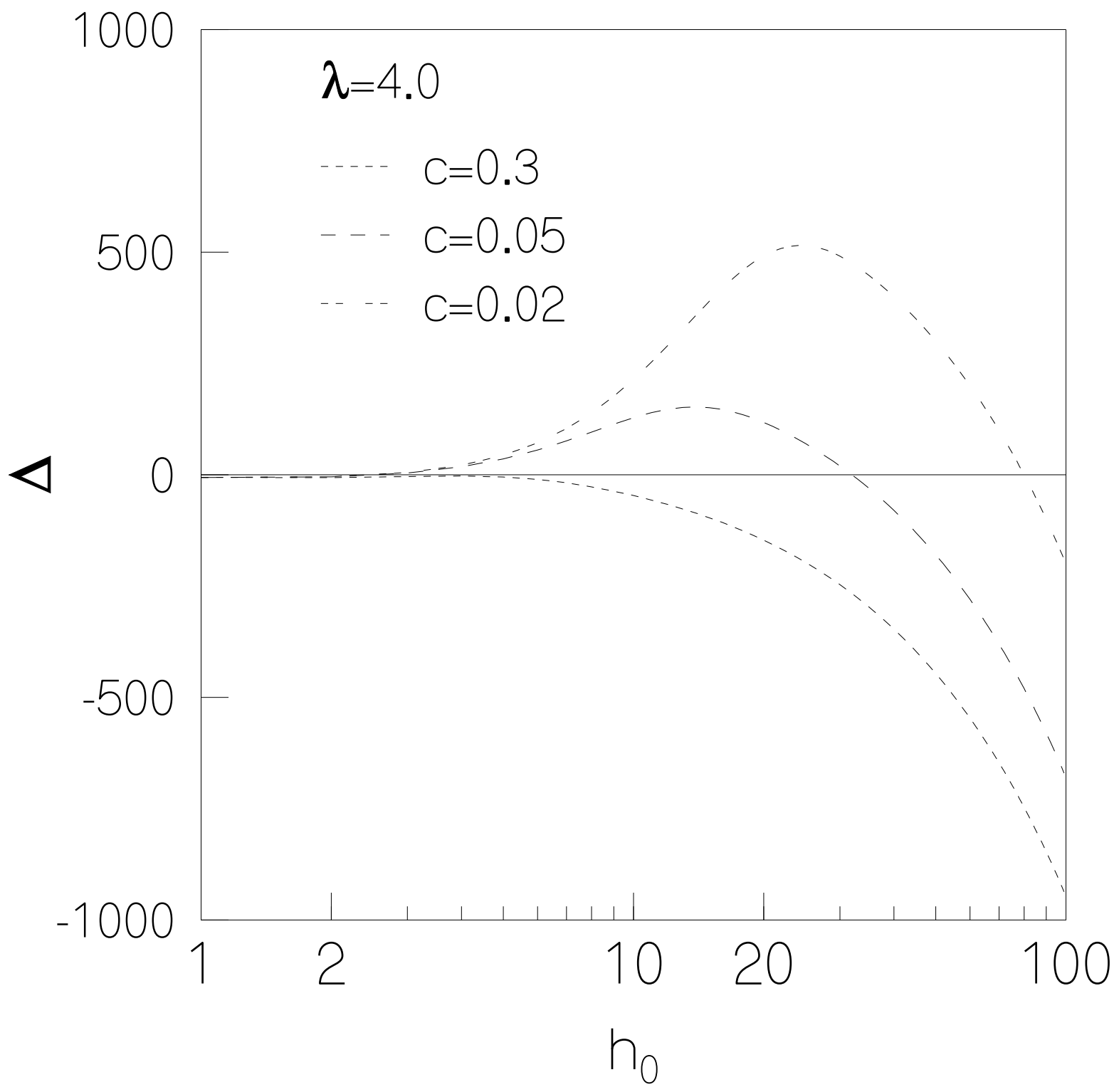




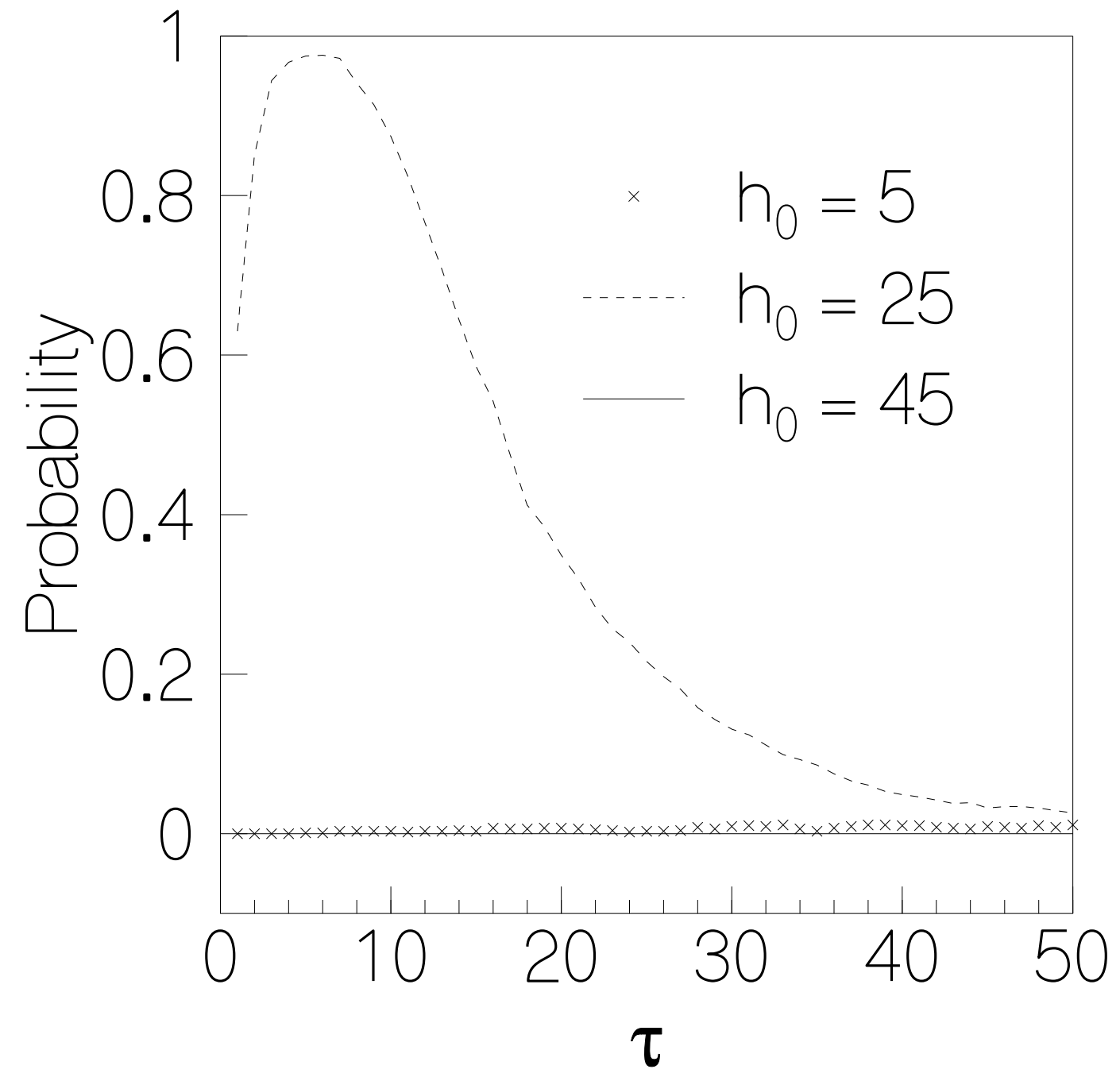




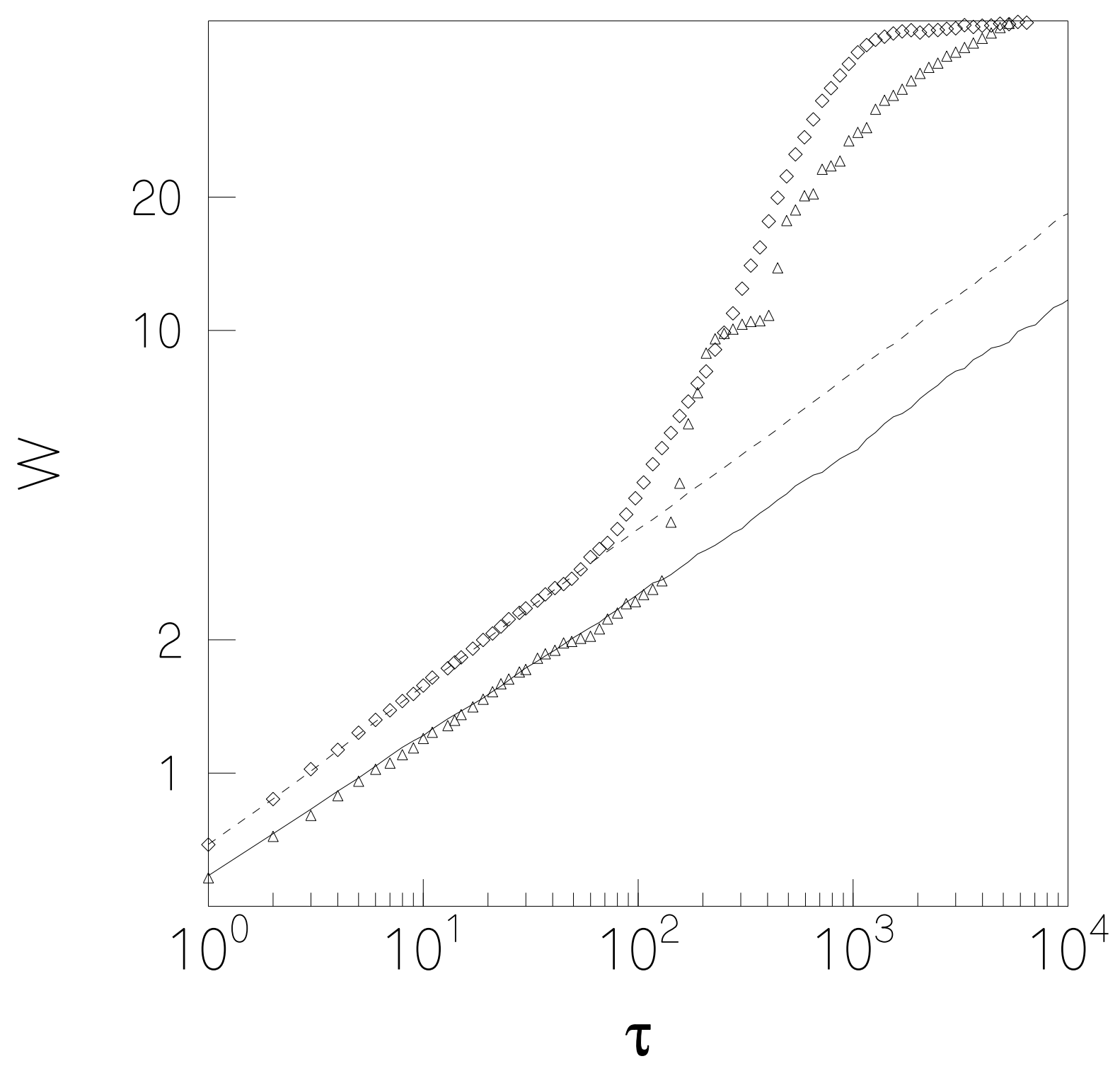




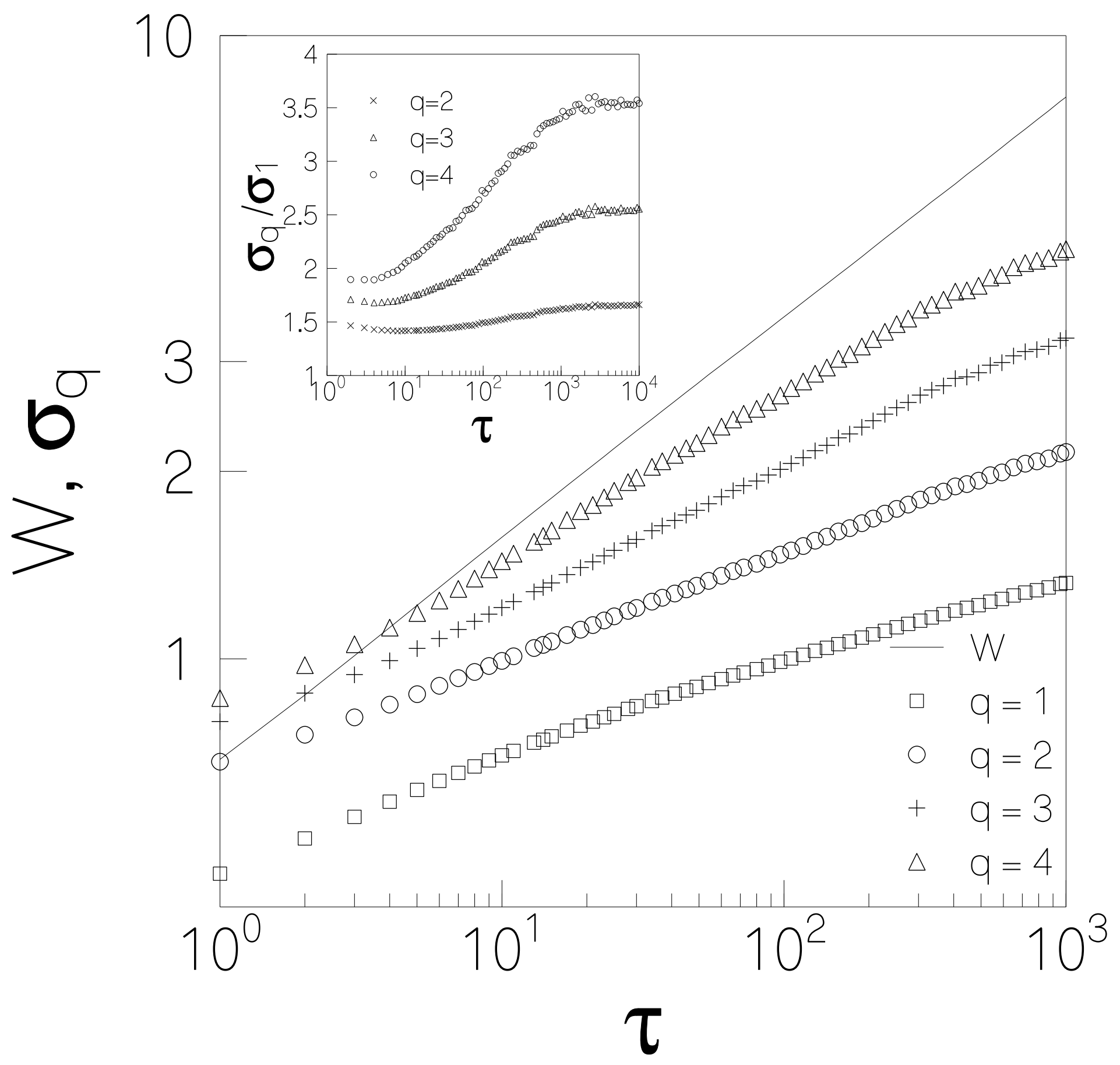




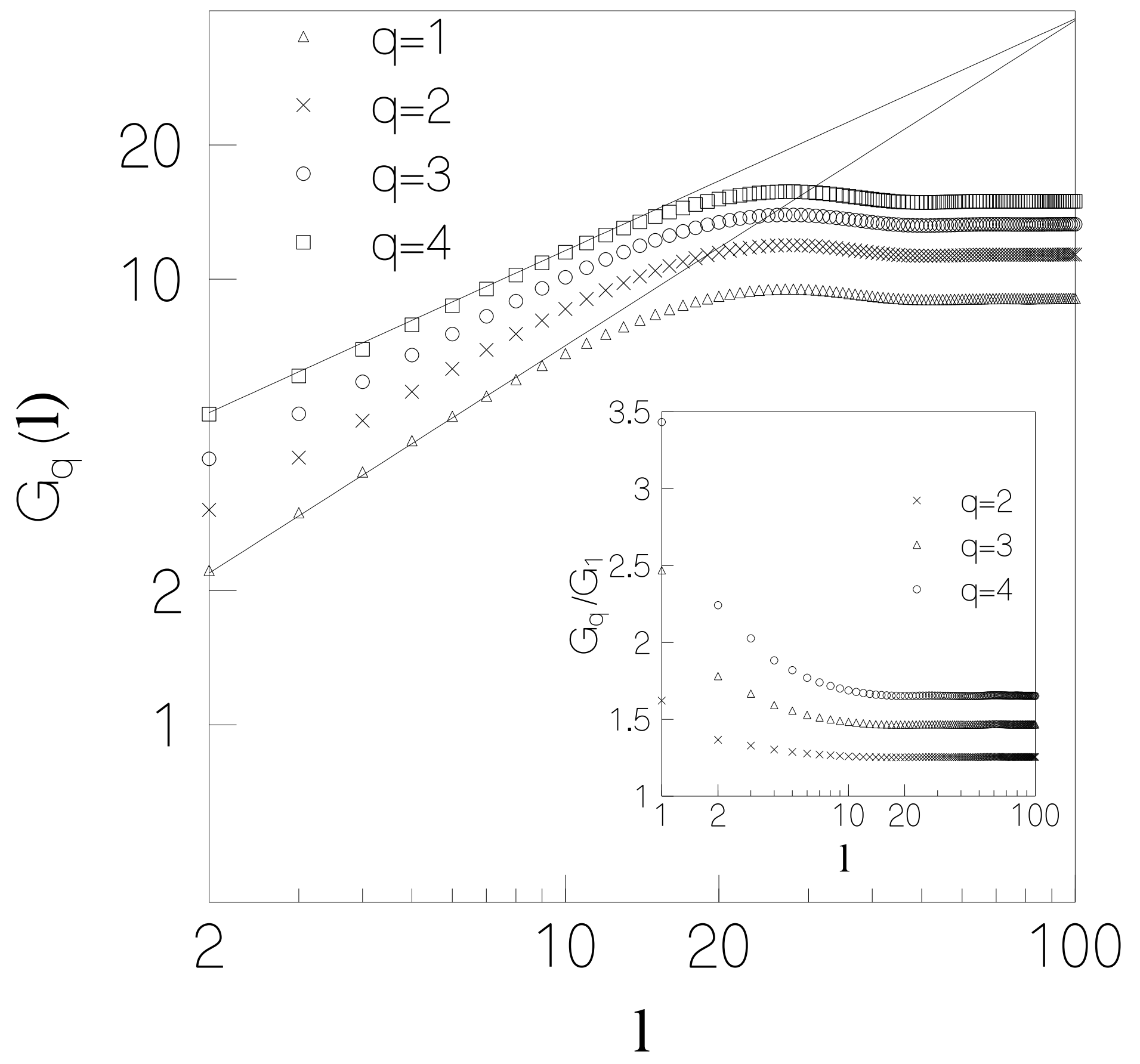




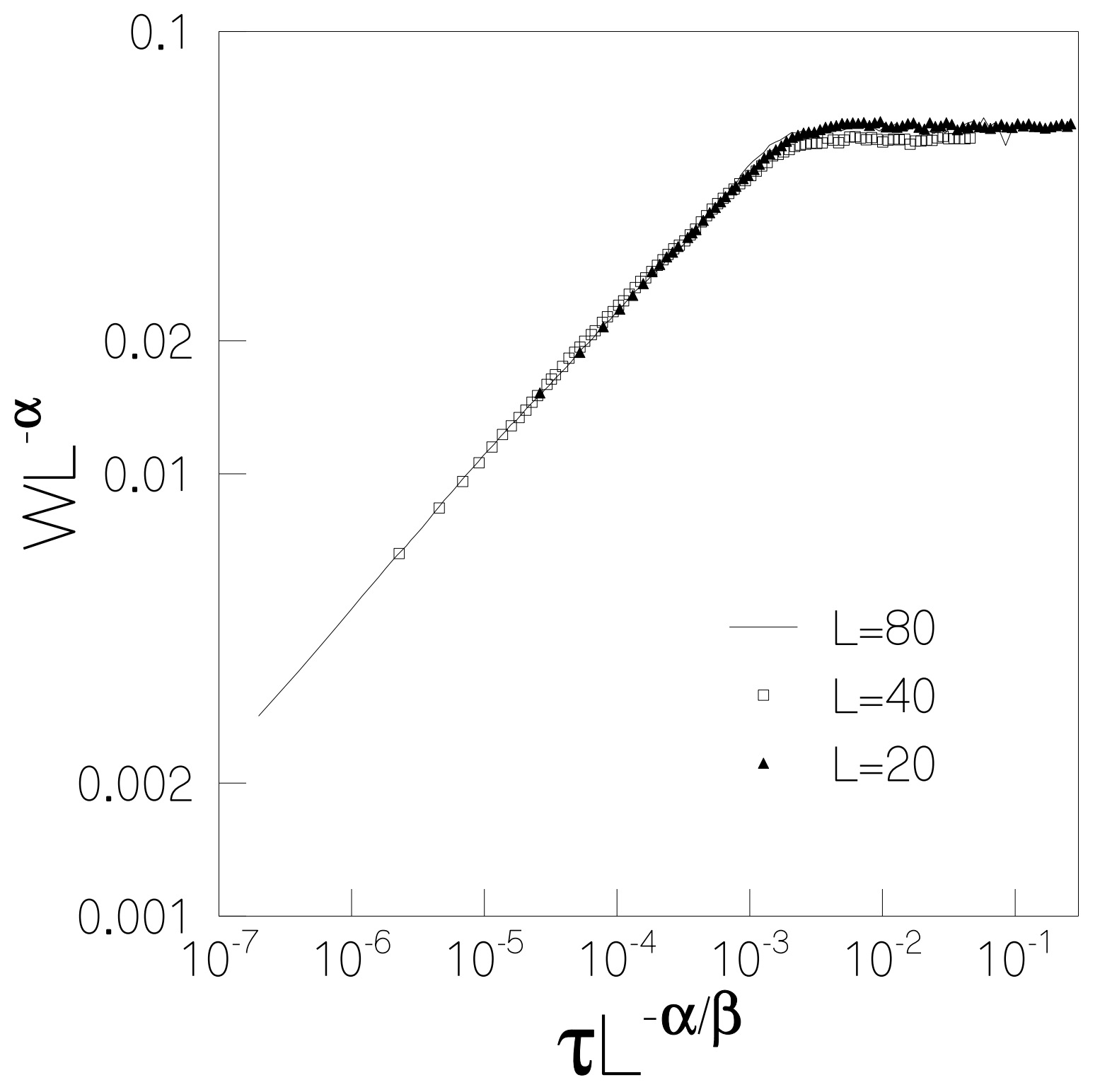




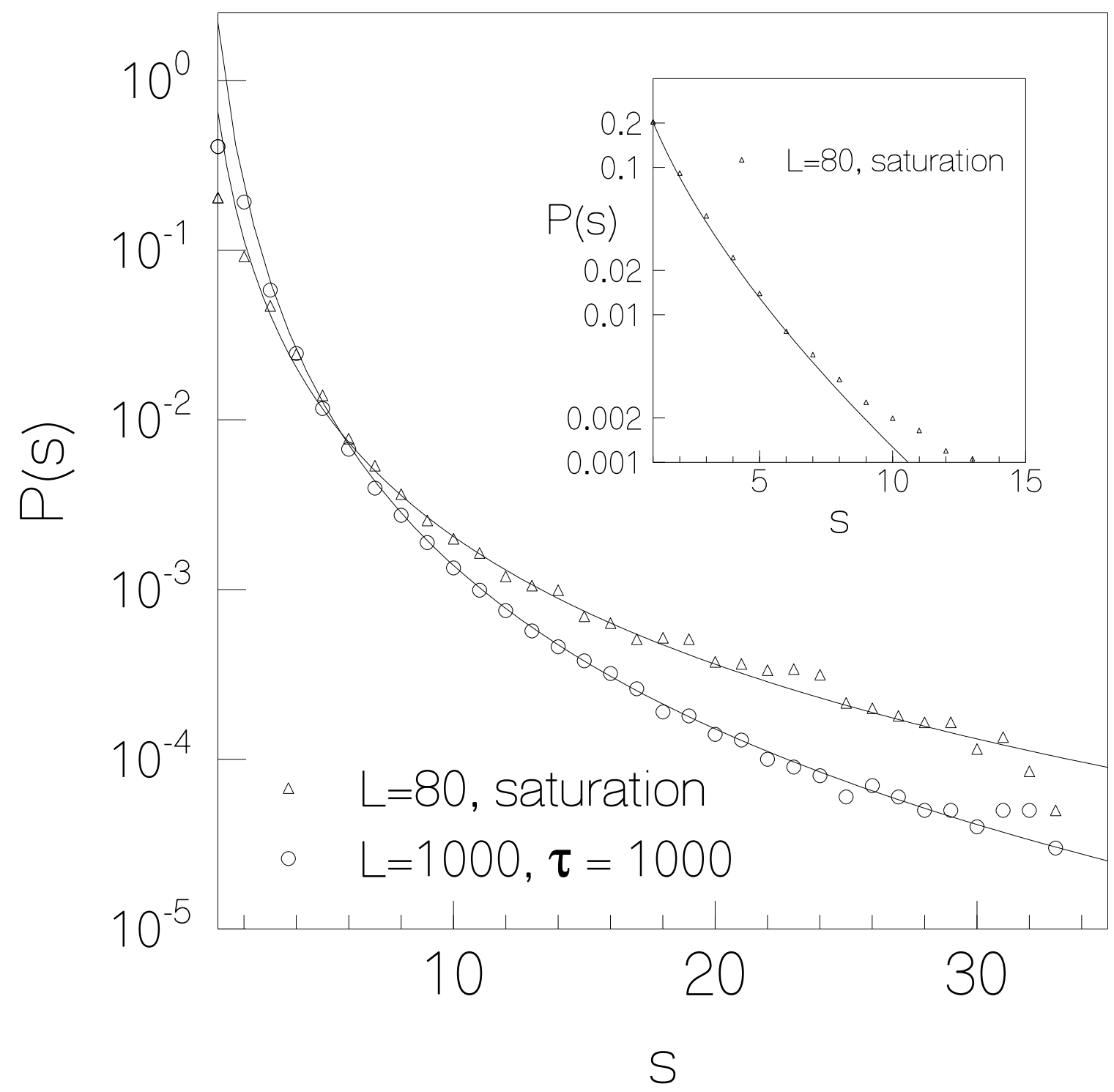




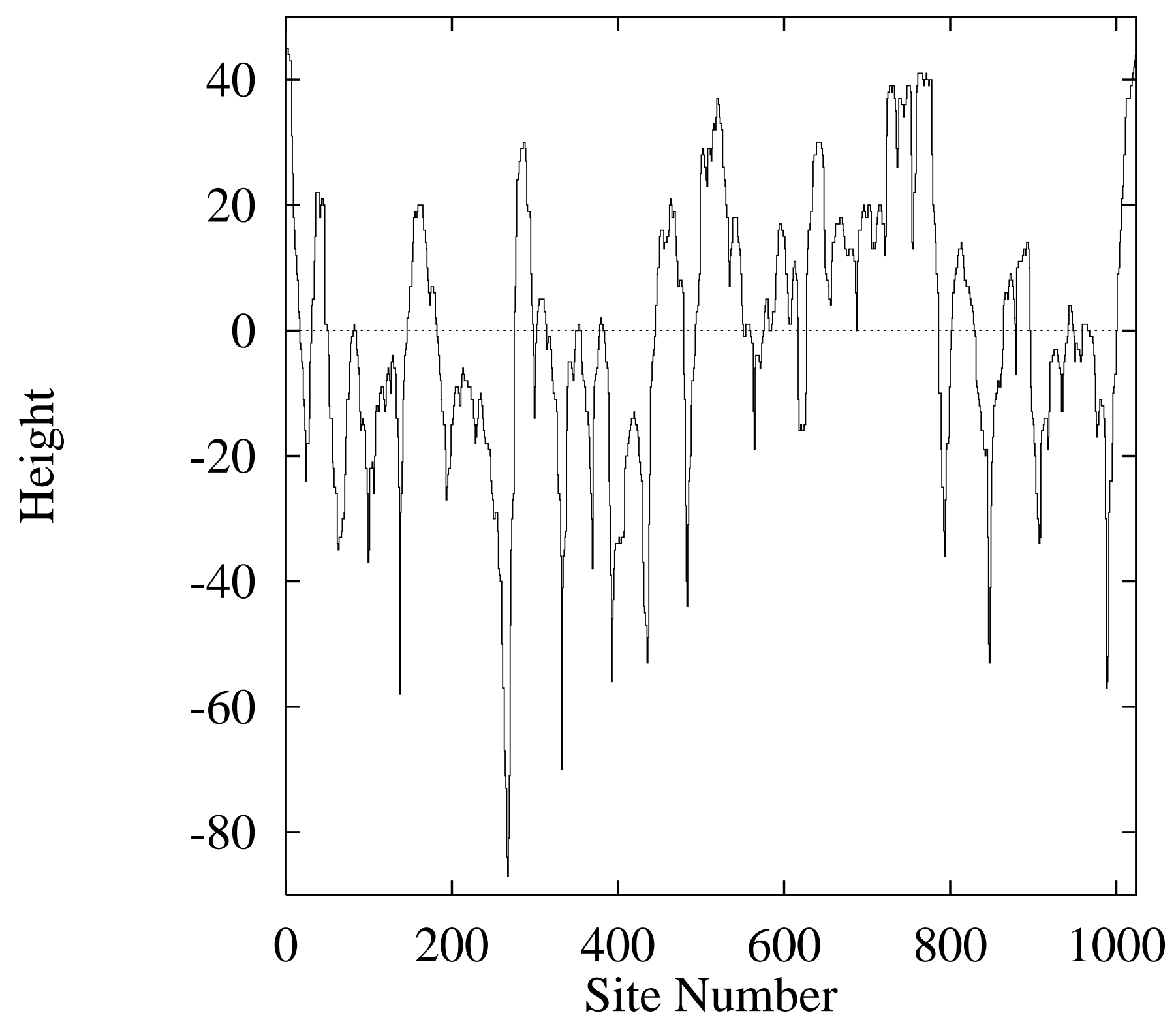




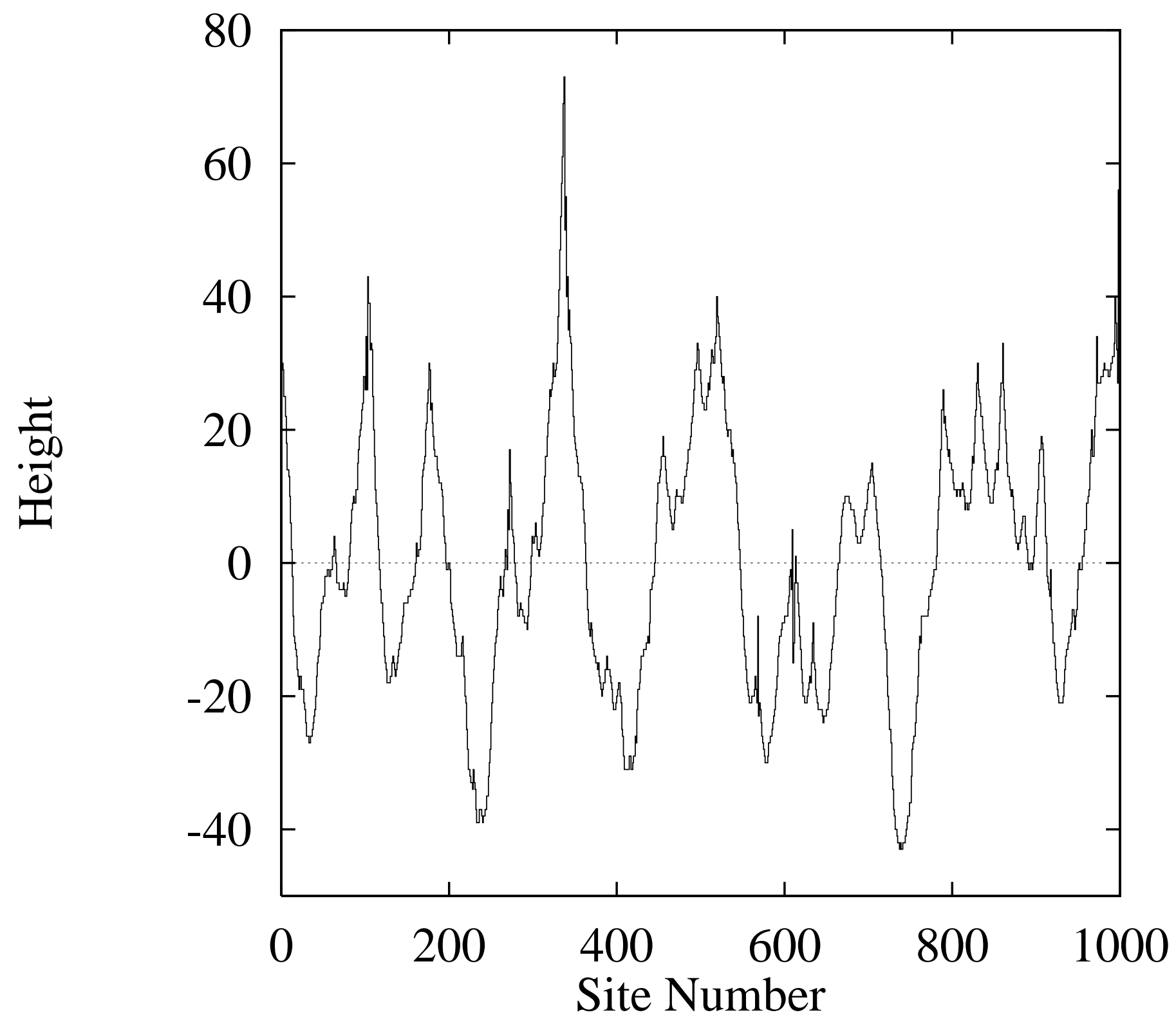




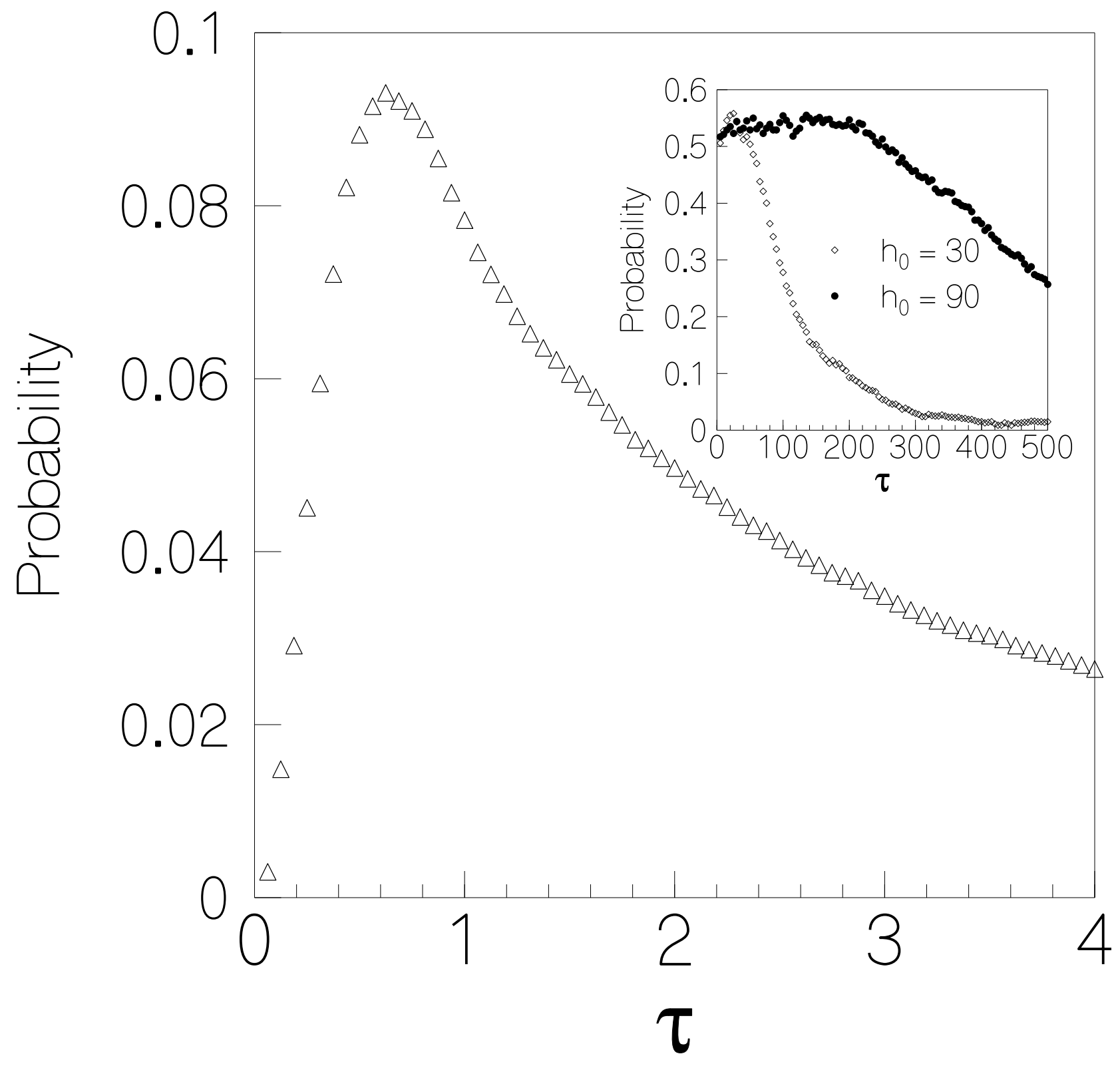




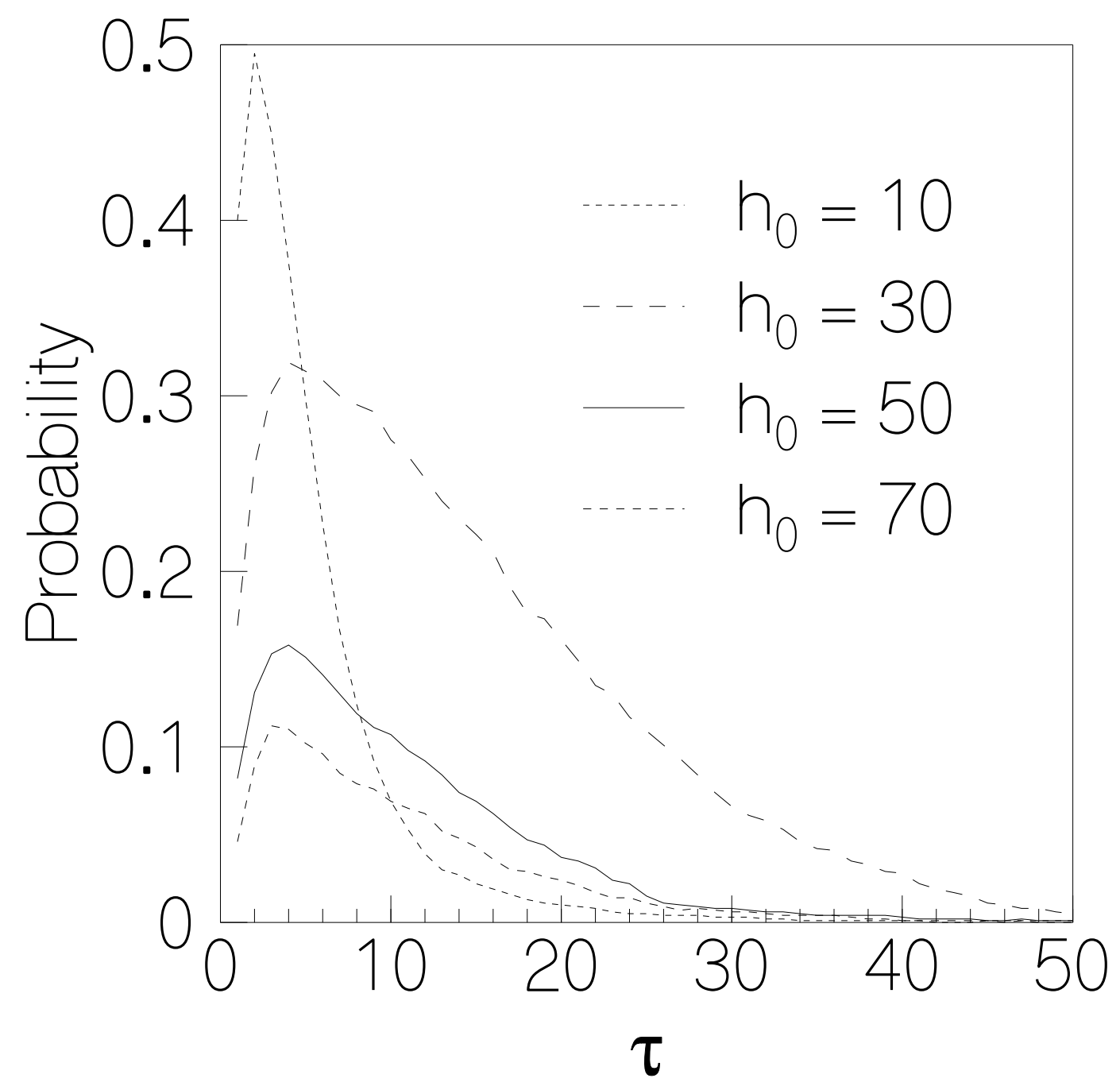




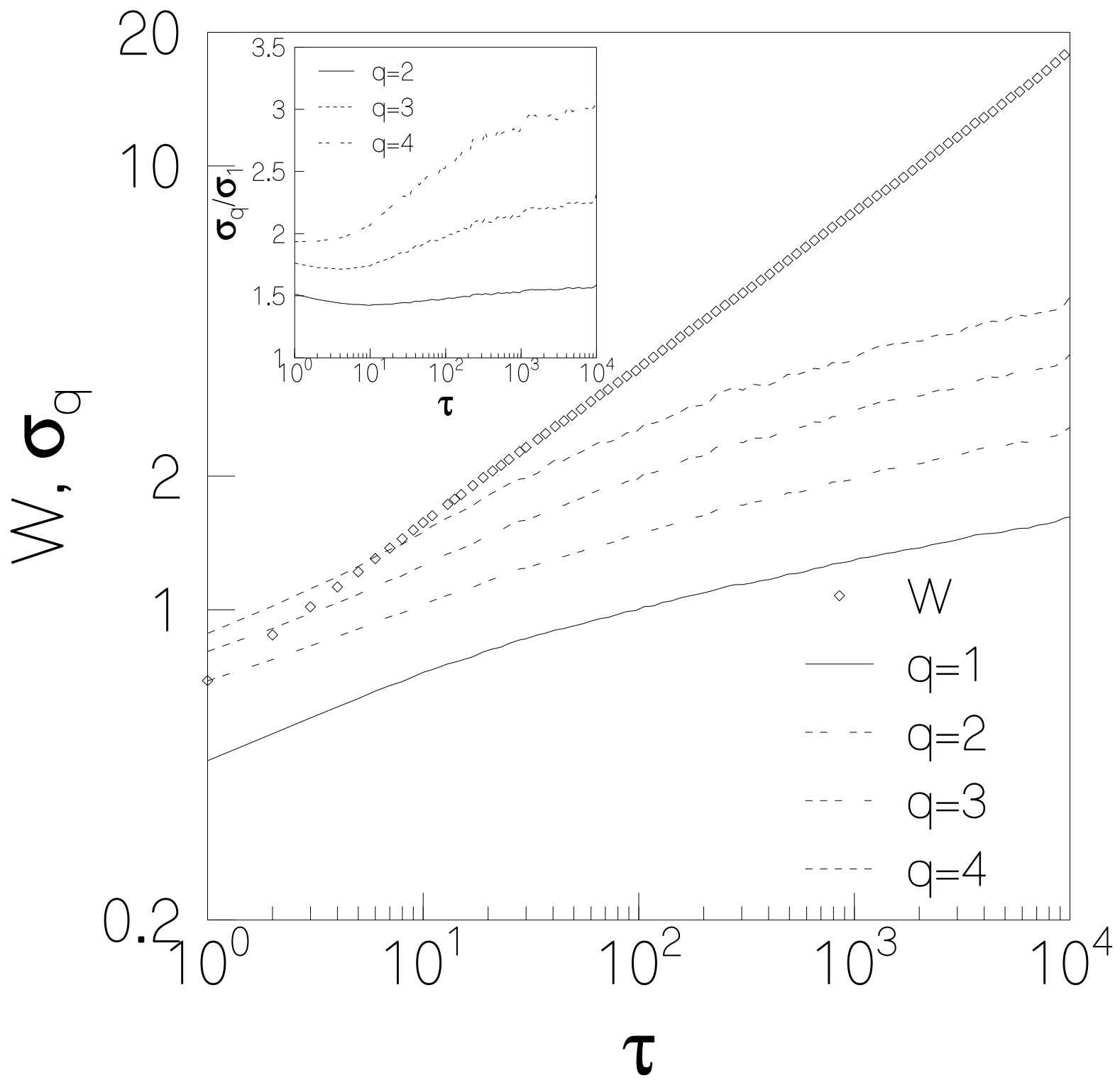




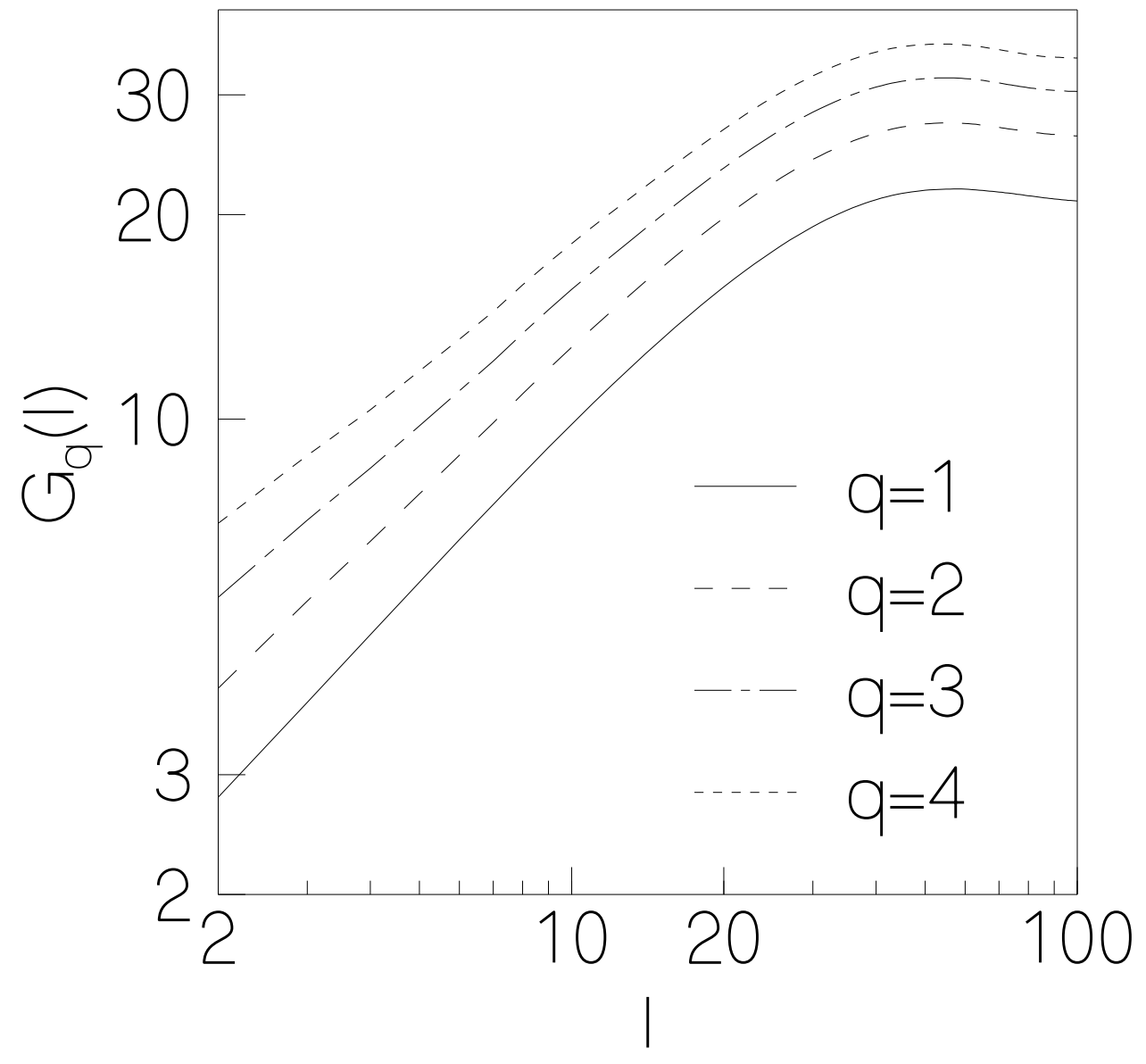




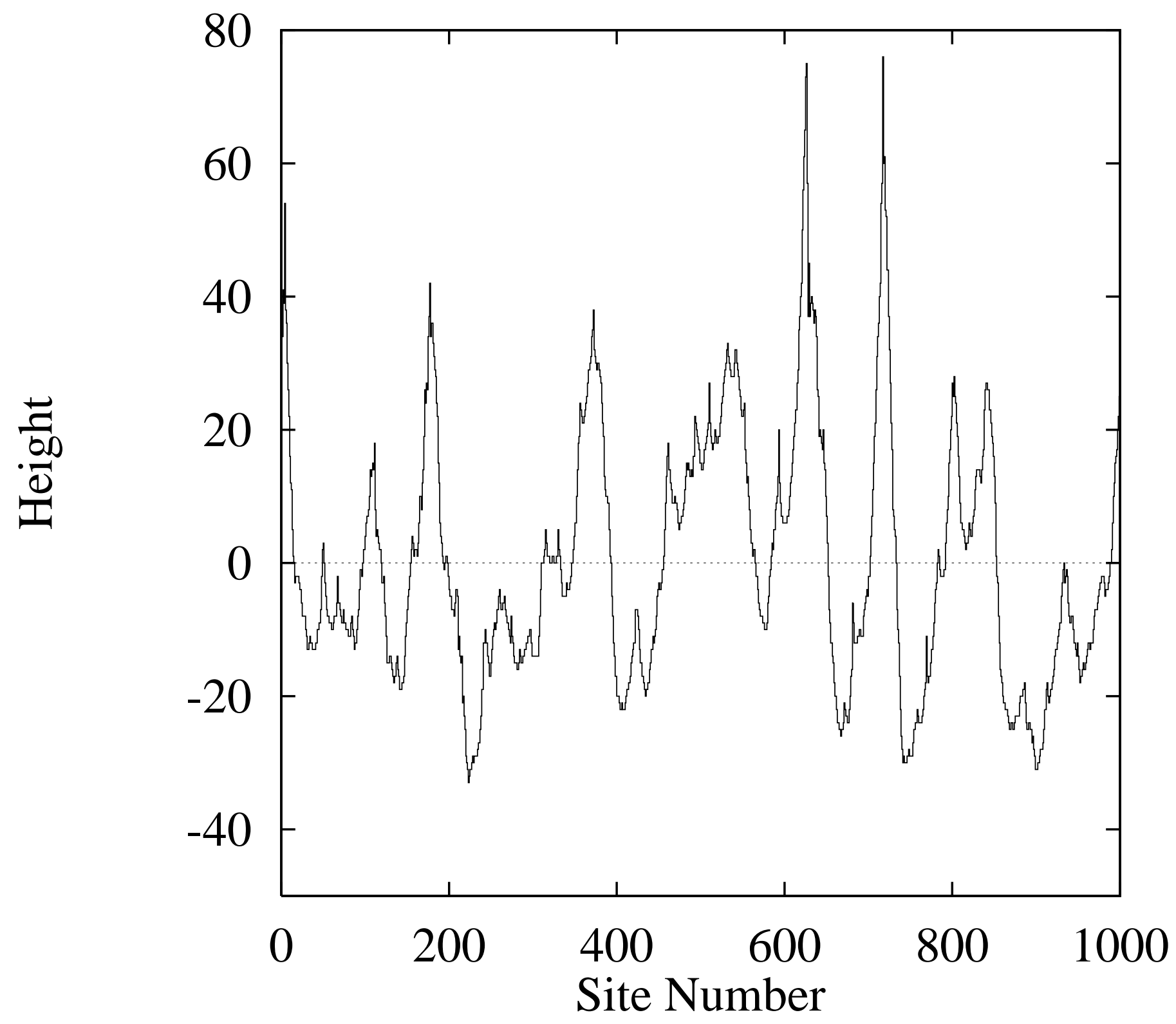

